\title{
Passer en revue quarante ans de Travail et Emploi
}

Reviewing 40 Years of Travail et Emploi

Thomas Amossé, Bruno Ducoudré, Christine Erhel, Arnaud Mias, Carine Ollivier, Camille Peugny, Laure Pitti, Géraldine Rieucau et Véronique Simonnet

\section{(2) OpenEdition}

1 Journals

Édition électronique

URL : http://journals.openedition.org/travailemploi/9036

DOI : 10.4000/travailemploi.9036

ISSN : 1775-416X

Éditeur

DARES - Ministère du Travail

Édition imprimée

Date de publication : 2 octobre 2019

Pagination : 7-42

ISSN : 0224-4365

\section{Référence électronique}

Thomas Amossé, Bruno Ducoudré, Christine Erhel, Arnaud Mias, Carine Ollivier, Camille Peugny, Laure Pitti, Géraldine Rieucau et Véronique Simonnet, "Passer en revue quarante ans de Travail et Emploi », Travail et Emploi [En ligne], 158 | 2019, mis en ligne le 01 novembre 2019, consulté le 09 décembre 2020. URL : http://journals.openedition.org/travailemploi/9036 ; DOl : https://doi.org/10.4000/ travailemploi.9036 


\title{
Passer en revue quarante ans de Travail et Emploi
}

\author{
Thomas Amossé, Bruno Ducoudré, Christine Erhel, \\ Arnaud Mias, Carine Ollivier, Camille Peugny, \\ Laure Pitti, Géraldine Rieucau, Véronique Simonnet
}

\begin{abstract}
Dans quelle mesure l'évolution des articles publiés depuis 1979 dans Travail et Emploi dessine-t-elle une histoire cohérente ? Pour essayer de répondre à cette question, nous proposons une analyse des articles parus dans la revue au cours de ces quatre décennies à partir de leurs titres et mots-clés. Pour rendre compte et éclairer cette histoire, trois types d'arguments sont mobilisés. Travail et Emploi étant initialement une revue de nature administrative, publiée par le ministère du Travail, l'évolution de ses articles est en partie liée aux soubresauts de l'actualité législative, des politiques publiques et de la production statistique. Elle peut par ailleurs pour partie tenir aux transformations du regard porté par les sciences sociales sur le travail et l'emploi à partir du moment où Travail et Emploi devient une revue résolument académique. Enfin, ces évolutions rendent également, et assez naturellement, compte des transformations réelles et profondes du travail et de l'emploi sur le terrain, dans la vie des travailleurs et des entreprises.
\end{abstract}

$\mathrm{L}$ 'allongement de la jeunesse constitue l'un des résultats majeurs de la sociologie des âges de la vie des années 1990 (GALland, 2000). Malgré tout, à 40 ans, il semble bien que la revue Travail et Emploi soit entrée dans l'âge adulte, sinon dans la maturité. Sans accorder à ce changement de décade plus de signification que nécessaire ${ }^{1}$, il nous a semblé opportun de nous livrer à l' exercice du bilan en nous extrayant quelques instants de la vie quotidienne nécessairement tumultueuse d'une revue pour porter un regard sur ces quarante premières années d'existence.

Si l'intention est louable, l'exercice n'est pas forcément aisé. Comment donner à voir l'évolution d'une revue créée en 1979, dont la nature a sensiblement évolué au cours du temps? Travail et Emploi est d'abord une revue portée par le Service des

\footnotetext{
* Membres du comité de rédaction. Leurs affiliations sont indiquées en deuxième de couverture.

1. Depuis les travaux de Laurent ThÉvENOT (1979), il est acquis qu'une certaine prudence est nécessaire dans l'analyse des catégories d'âge.
} 
études et de la statistique (SES) du ministère du Travail, dont le chef signe les éditoriaux et qui fonctionne sans comité de rédaction jusqu'en 1986. En 2019, elle est une revue académique pluridisciplinaire de recherche en sciences sociales qui fonctionne selon les règles rigoureuses de toute revue scientifique (encadré). De la même manière, le regard porté par les différentes disciplines des sciences sociales sur le travail et l'emploi et les méthodes d'analyse que celles-ci utilisent ont fortement évolué au cours des quarante années passées sous revue. Dépendant de l'actualité scientifique, les thématiques abordées comme les catégories mobilisées se sont sensiblement modifiées : certaines, présentes sur toute la période, ont simplement fluctué ; mais d'autres ont pratiquement disparu alors que de nouvelles émergeaient. Enfin, et de façon centrale, l'objet même de la revue - le travail et l'emploi - s'est profondément transformé. Il figure parmi les réalités sociales qui ont été les plus affectées sur la période par les soubresauts de la conjoncture économique et la marche des politiques publiques. Il est par ailleurs traversé par d'importantes mutations. Dans l'éditorial du premier numéro, le ministre du Travail de l'époque, Robert Boulin, assignait à la revue la mission de documenter les « problèmes de travail et d'emploi [qui] ont pris depuis quelques années une dimension nouvelle » en raison d'une « situation économique de crise caractérisée par son ampleur et sa durée ». Tout au long des quatre décennies qui se sont écoulées depuis, le travail et l'emploi ont ainsi constitué des sujets de premier plan dans une bonne partie du débat public.

Pour toutes ces raisons, à l'heure du bilan, il n'est pas évident d'identifier les fils à tirer ni même les mailles, ou unités de mesure, à utiliser. Néanmoins, pour tenter de relever le défi et d'objectiver un certain nombre d'évolutions, nous proposons dans cet article des éléments d'analyse à partir de l'examen systématique de l'évolution des titres et mots-clés du millier d'articles publiés depuis 1979.

ENCADRÉ

\section{Quarante ans de vie de Travail et Emploi}

En parcourant les numéros de la revue depuis sa création et en échangeant avec des personnes qui y ont joué un rôle clé, se profile une image de ce qu'a été, au cours de ses quarante années, la vie de la revue Travail et Emploi : sa programmation, ses acteurs, son public, ses coulisses.

\section{9-1986 : les premiers pas de Travail et Emploi}

Travail et Emploi, dont le premier numéro sort au troisième trimestre 1979, est la deuxième revue scientifique créée dans le giron des ministères sociaux, après la Revue française des affaires sociales en $1967^{1}$. Cette création, au sein du Service des études et de la statistique (SES) du ministère du Travail, est marquée par la volonté de construire un espace de réflexions spécifiquement consacré au travail et à l'emploi, dans un contexte de crise durable, afin de fournir à l'administration et aux décideurs des éléments de compréhension

1. La Revue française du Travail, créée en 1946, est devenue en 1967 Revue française des Affaires sociales (RFAS). Sur l'histoire de la RFAS, voir l'article de Vincent VIET (2006). 
qui facilitent leur action et de multiplier l'information du public : tel est en substance le message de Robert Boulin, ministre du Travail et de la Participation, dans l'éditorial du premier numéro. La force de cet éditorial est d'exposer ce qui fera le cœur des premières années de la revue : l'émergence de travaux qui, à côté des analyses macro-économiques de l'emploi et du chômage et des données administratives, prennent appui sur des enquêtes de terrain et de nouvelles enquêtes statistiques, et/ou qui s'attachent à tracer les parcours biographiques des personnes (Amossé, 2020, à paraître) ; dans une optique d'emblée pluridisciplinaire (économie, sociologie, droit, etc.), ces travaux mettent en évidence les conditions de travail, les nouveaux modes de gestion des entreprises, participent à la compréhension des « comportements nouveaux » des chômeurs, des femmes, des jeunes, et à celle des changements du droit du travail.

Ces débuts sont marqués par la nécessité de faire vivre et de faire connaître la revue, qui fonctionne alors sans comité de rédaction et sans normes précises de publication, de recueillir et de susciter des articles et des synthèses des travaux auprès des membres du SES, du Centre d'études de l'emploi (CEE), du Centre d'études et de recherches sur les qualifications (Céreq), de l'Agence nationale pour l'emploi (ANPE), etc. Pour illustrer cette époque, Martine Lurol, en charge de la rédaction et du secrétariat de 1981 à $1995^{2}$ évoque un « travail artisanal, un peu du bricolage, mais qui tenait quand même la route ».

\section{7-1994 : l'adolescence? Une revue qui s'ouvre et qui s'affirme}

Une deuxième période s'ouvre dans l'histoire de la revue avec, en 1986, la mise en place d'un comité de rédaction, dont les membres sont issus de l'administration et du monde académique, des premiers critères établis pour sélectionner des propositions d'articles, désormais plus nombreuses à être soumises de façon spontanée. Dès 1988, le contenu s'enrichit, avec des articles rassemblés dans une rubrique «Études » ou « Dossier » lorsqu'ils portent sur un même thème ${ }^{3}$. C'est également l'âge de l'ouverture à l'international, concrétisée par une mise en visibilité des publications sur des pays étrangers ou présentant des comparaisons internationales, réunies dans une rubrique « Observatoire international », et par la reproduction des sommaires des revues d'un «Club de revues étrangères », parties prenantes du colloque international sur l'évolution des formes d'emploi ${ }^{4}$, qu'organise Travail et Emploi à Paris en novembre 1988, et dont la publication de contributions marquera l'anniversaire des 10 ans (TRAVAIL ET EMPLOI n ${ }^{\circ} 39,1989$ ). La revue affirme par ailleurs ses fondamentaux, avec des travaux menés sur des enquêtes émanant de l'administration, des informations générales dans la rubrique « En bref » sur les enquêtes, les études, les colloques ou la conjoncture, à une époque où le support papier est le principal mode de communication.

L'adolescence est aussi le temps des mutations corporelles. En 1991, la maquette connaît son premier changement notable, avec une couverture passant d'un mauve layette

2. Françoise Murillo a également occupé cette fonction au cours de cette période, Frédérique Trimouille dans la seconde moitié des années 1990.

3. Un « dossier spécial » consacré au temps de travail, a toutefois été publié dès le troisième numéro, cf. TRAVAIL ET EMPLOI n ${ }^{\circ}$ 3, 1980 (notée ainsi, la référence renvoie à un numéro thématique de la revue).

4. Les revues sont : Lavoro e Diritto (Italie); Arbeit und Recht (Allemagne); The Comparative Labor Law Journal (États-Unis) ; The Industrial Law Journal (Grande-Bretagne) ; The International Journal of Comparative Labour Law and Industrial Relations (Grande-Bretagne); Relaciones Laborales (Espagne); The Review of Japan Institute of Labour (Japon). 
à un jaune pour le moins voyant, assorti d'un graphisme avec des losanges : cette mue ne dure (heureusement) que deux ans et, en 1994, la couverture s'assagit en optant pour un logo plus discret mais surtout pour ce qui deviendra sa signature visuelle : le bleu, l'orange et la vague montante. Pour ses 20 ans, elle abandonnera complètement le logo pour ne conserver que le bleu, l'orange et la fameuse vague qui font sa marque aujourd'hui encore.

\section{4-2009 : une jeunesse à la Dares}

En 1994, la revue, désormais éditée par la Direction de l'animation de la recherche, des études et des statistiques (Dares) créée en 1993, aborde une nouvelle phase. À côté des articles, rassemblés ou non dans des dossiers, qui font le cœur de la revue, les notes de lecture, rarissimes jusque-là, deviennent plus fréquentes et la revue publie des pages débats, notamment autour d'ouvrages marquants. Son ADN est toujours le même : pluridisciplinaire, avec l'économie, la sociologie, l'ergonomie et le droit, elle s'ouvre à l'histoire et affiche une volonté accrue de faire le lien avec le monde de la recherche. «Beaucoup de choses se passaient alors à Travail et Emploi, qui était le réceptacle de tous les grands débats et des recherches », rappelle Dominique Méda, qui fut cheffe de la Mission animation de la recherche, avant que Dominique Goux ne la remplace. Toutes deux ont alors animé le comité de rédaction composé d'une vingtaine de membres, où la parité entre administratifs et académiques (et entre femmes et hommes) est respectée. Ce comité travaille sous l'égide du directeur de la Dares, Claude Seibel (1993-2000), puis de sa directrice, Annie Fouquet (2000-2005). L'ouverture internationale se renforce, avec la participation dans le comité de rédaction de chercheurs et universitaires d'autres pays européens.

L'anniversaire de ses 25 ans est l'occasion d'éditer un DVD sur les cent premiers numéros et de publier un numéro au sein duquel des auteurs reviennent sur les articles qu'ils ont écrits dans la revue, « avec un regard embrassant le passé et l'avenir » (Introduction, TRAVAIL ET EMPLOI n ${ }^{\circ} 100,2004$, p. 6). De ces années, Marie Wierink, qui fut secrétaire de rédaction entre 1998 et 2003, garde le souvenir d'une activité dense et passionnante, mais aussi, là encore, d'un travail marqué par son caractère artisanal : à chaque numéro, l'ouvrage est remis sur le métier.

\section{9 et après : l'âge de la maturité ?}

À partir de 2009, la revue entre dans un «nouveau cycle », pour reprendre l'expression d'Antoine Magnier, directeur de la Dares de 2005 à 2014, et qui met en œuvre un changement de gouvernance. Concrètement, en 2009, le comité de rédaction est resserré et refondé autour de sept ou huit membres, tous issus du monde académique, avec d'abord Pierre Ralle comme rédacteur en chef (2009-2010). Le comité fonctionne de façon totalement indépendante de l'administration, prenant appui sur une évaluation toujours collégiale des articles mais qui recourt dès lors systématiquement à des rapports de lecture commandés à des chercheurs extérieurs.

Ce changement est marqué par le souhait de rapprocher davantage encore la revue des canons académiques, alors que la concurrence entre les revues s'accroît. Il s'inscrit également dans le contexte d'une demande de travaux sur l'évaluation des dispositifs des politiques publiques, évaluation qui, sans être nouvelle, se complexifie, s'enrichit de nouvelles données et d'une réflexion poussée sur les méthodes. Cette orientation ne signifie pas un abandon de ses premières amours : laissant une large place aux articles mobilisant des enquêtes de terrain (monographies d'entreprises ou de secteurs, récits ou observations des situations de travail) ou des données statistiques, la revue maintient son orientation 
pluridisciplinaire, en associant notamment sur les thématiques fortes du moment (les risques psychosociaux, la discrimination syndicale) des points de vue d'historiens, juristes, politistes ou gestionnaires en plus des regards de sociologues et économistes, plus classiques dans ses pages.

Avec le soutien du directeur (Antoine Magnier, de 2005 à 2014) et des directrices successives de la Dares (Françoise Bouygard de 2014 à 2016, Selma Mahfouz depuis), la revue est pilotée par Thomas Amossé (de 2010 à 2018), puis par Camille Peugny et Géraldine Rieucau, Micheline Léger (2004-2012) et Magali Marcille depuis 2012, étant à l'œuvre au secrétariat de rédaction. À l'approche de la quarantaine, l'envie d'une nouvelle jeunesse se fait sentir : la revue fait donc peau neuve en 2015 avec un nouveau format et une nouvelle maquette. Elle alterne dossiers thématiques et numéros varia et publie, tous les deux ans, un numéro spécial avec une sélection d'articles en anglais issus des numéros antérieurs. Gageons que Travail et Emploi a de belles pages devant elle à écrire.

\section{Une analyse des articles à partir de leurs titres et mots-clés}

Au total, si l'on excepte les recensions d'ouvrages et certaines pages de la rubrique « Théories et débats », 980 articles ont été publiés dans Travail et Emploi sur la période 1979-2018 (du numéro 1 au numéro 154), chacun étant décrit par des mots-clés. Dans la mesure où les mots-clés utilisés ont changé au cours du temps et où leur nombre est extrêmement variable d'un article à l'autre, il était difficile d'opérer une classification des articles selon cette indexation par mots-clés. Nous avons donc décidé d'établir un plan de classement thématique afin d'affecter à chaque article un ou plusieurs thèmes. En nous appuyant sur les classifications du Bureau international du travail (BIT), de la revue Liaisons sociales et du Journal of Economic Litterature (JEL), nous avons construit une classification en 21 thèmes, que nous avons ensuite regroupés en 13 grands thèmes à des fins de lisibilité (tableau). Nous avons fait en sorte que tout en rendant compte de la variété des disciplines publiées par la revue (sociologie, économie, droit, histoire, gestion, etc.), cette classification conserve le caractère transversal des thèmes retenus.

Nous avons ensuite affecté manuellement 1, 2, 3 ou 4 thèmes de la classification à chaque article en fonction de son titre et de ses mots-clés. Nous avons ainsi la liste des articles avec les thèmes de classification associés. Afin que tous les articles aient un poids identique dans la classification finale, nous avons attribué à chacun d'entre eux une pondération égale à l'inverse du nombre de thèmes qui lui ont été associés. On peut ainsi comptabiliser chaque année le nombre d'articles consacrés à chaque thème. Étant donné que le nombre total d'articles publiés varie d'une année sur l'autre, et pour ne pas surpondérer les années où ce nombre est plus important, nous avons rapporté le nombre d'articles consacrés à un thème une année donnée au nombre total d'articles publiés cette même année. La part de chaque thème dans l'ensemble des articles publiés tout au long des quarante dernières années est représentée dans le graphique. Les thèmes regroupés au sein d'un même grand thème sont accolés pour pouvoir comparer plus facilement leurs évolutions. 
TABLEAU - Plan de classement des mots-clés des articles parus dans Travail et Emploi (1979-2018)

Thèmes (21, regroupés en 13 grands thèmes)

Relations de travail, syndicalisme (négociations et conventions collectives, dialogue social, conflits du travail et mouvements sociaux, institutions représentatives du personnel [IRP], organisations d'employeurs et de travailleurs, participation des travailleurs, relations de travail, syndicalisme)

Politique et administration publique (politique de l'emploi [création, promotion], politique du travail, politique sociale, évaluation, administration publique et du travail), protection sociale (indemnisation du chômage, service de l'emploi, minima sociaux, cotisations sociales, systèmes de retraite, pauvreté et conditions sociales, aide sociale, sécurité sociale, services sociaux)

Conditions et organisation du travail (milieu et conditions de travail, organisation du travail, qualité de la vie de travail, psychologie sociale et sociologie), santé (sécurité et santé au travail, accidents du travail et maladies professionnelles, incapacité et handicap, risques professionnels, satisfaction)

Formes d'emploi (indépendants, salariés, statuts particuliers [informel, fonction publique], contrats de travail), temps de travail (durée du travail, temps partiel, congés divers, heures supplémentaires)

Ressources humaines et gestion du personnel (réaffectation et mutation, recrutement et sélection, conditions d'emploi, analyse des tâches, appréciation des performances, rupture de contrat, cessation de la relation de travail), mobilités et trajectoires (carrières et trajectoires, mobilité professionnelle, mobilité géographique)

Égalité et diversité (inégalités, discrimination, âge, genre, groupes démographiques, migration), famille (conciliation vie familiale/vie professionnelle, enfants)

Secteurs d'activité, professions (métiers, activités)

En pourcentage

des articles parus

12

11,7

(dont 5,7 et 6)

10,3

(dont 7,1 et 3,2)

10,2

(dont 5 et 5,2)

$\mathbf{9 , 2}$

(dont 7,2 et 2)

8,9

(dont 7,7 et 1,2)

8,1

(dont 4,9 et 3,2)

Capital humain et formation (qualification, compétences, productivité, éducation, 8 apprentissage, insertion professionnelle, formation professionnelle, expérience, ancienneté, déclassement), rémunération et coût du travail (salaires, structure salariale, coût du travail, épargne salariale)

International (comparaisons, pays)

Économie et conjoncture (crise, croissance, emploi-activité-chômage, macro-économie, structure de la main-d'œuvre)

Entreprise (employeurs, entrepreneurs et dirigeants, gestion et gouvernance, aides aux entreprises, actionnariat, association-organisation-autogestion, équipement et technologie), flux d'emplois (création, destruction et transfert d'entreprises ou d'emplois, emplois vacants, demande de travail)

Droit (droit au et du travail, droits économiques et sociaux, législation et normes du travail)

Histoire

Note : les thèmes sont ordonnés selon leur fréquence d'occurrence dans les articles.

Source : ensemble des articles parus dans la revue Travail et Emploi (1979-2018, du numéro 1 au numéro 154 ; n = 980). 

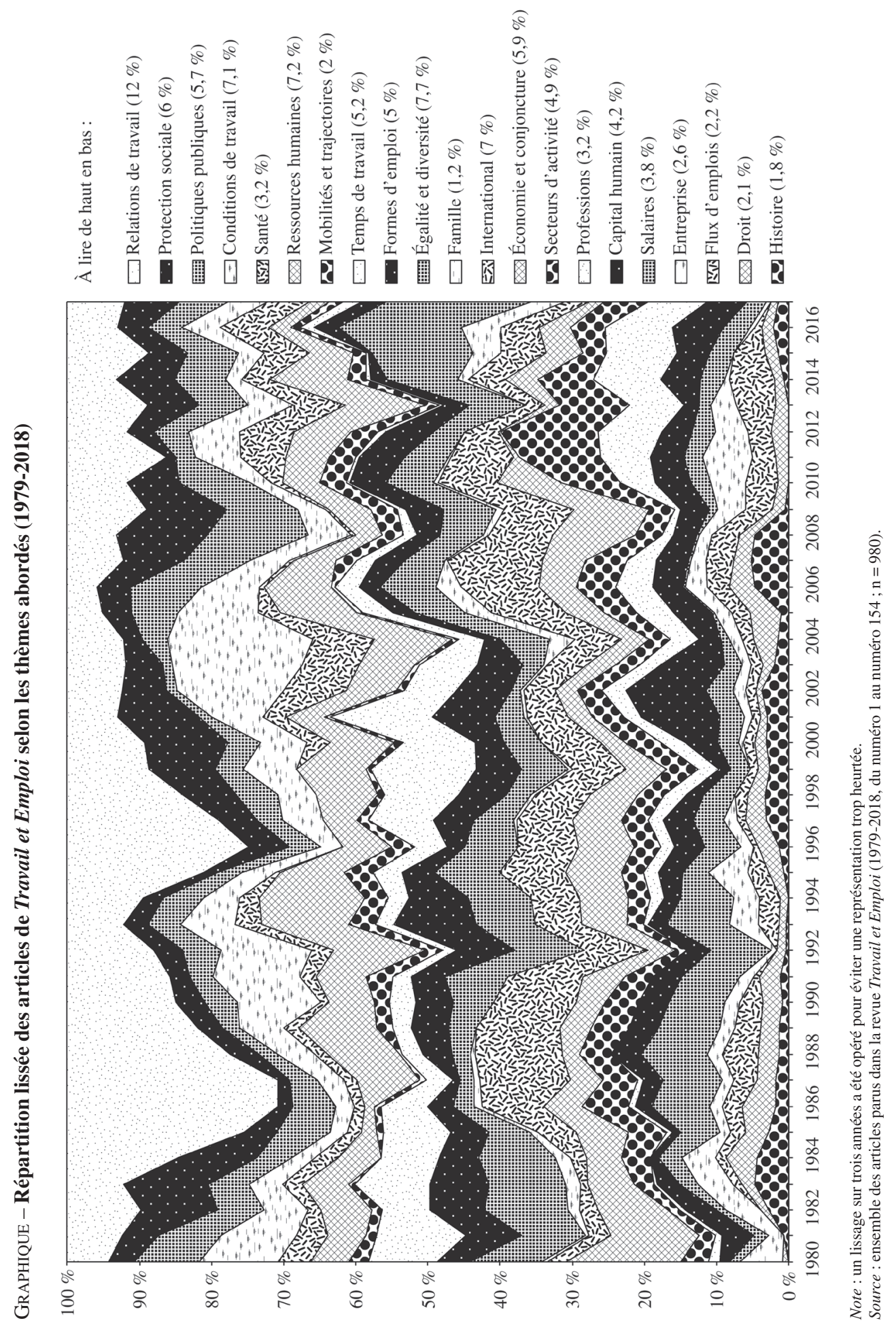
Les calculs réalisés dépendent bien sûr du plan de classement que nous avons retenu, mais on peut en tirer quelques enseignements : tout d'abord, le poids élevé de la thématique des relations de travail et du syndicalisme (12\%), notamment dû à un grand nombre d'articles parus sur ce thème au cours des deux premières décennies d'existence de la revue. Ensuite, la thématique « politique et administration publique » $(11,7 \%)$, en particulier les dispositifs visant à lutter contre le chômage, occupe une place notable dans la revue entre la fin des années 1990 et 2010. Celle des conditions de travail (incluant l'organisation du travail et la santé, 10,3\%) a quant à elle une importance plus cyclique, puisqu'elle rythme les publications de la revue au début des années 1980 et 1990, au milieu des années 2000 et dans la dernière décennie (grâce au poids croissant pris par les articles concernant la santé au travail). Les formes d'emploi et le temps de travail, dont la présence diminue à partir des années 2000, complètent le quatuor de tête des thématiques les plus fréquentes. Viennent ensuite les ressources humaines et les mobilités professionnelles, l'égalité, la diversité et la famille (thématique qui inclut les inégalités et la discrimination, notamment selon le genre), les secteurs d'activité et les professions. Au rang des surprises, la relativement faible présence d'articles traitant la question de la formation d'une part - ce qui peut tenir à la création de la revue Formation Emploi en 1983 -, et des salaires d'autre part, abordés néanmoins via la négociation salariale ou l'analyse du coût du travail. La dimension internationale de la revue concerne quant à elle $7 \%$ des articles publiés.

Ces tendances traduisent la capacité de la revue à porter ces thèmes tout au long de ces quarante années d'existence, de même que sa sensibilité aux réformes phares du marché du travail, à l'évolution des disciplines et aux transformations du travail et de l'emploi. Ces trois dimensions sont développées dans la suite de l'article.

\section{Un reflet de l'actualité législative, des politiques publiques et de la production statistique}

Travail et Emploi est de fait une revue dont la création est portée par le ministère du Travail puis soutenue par la Dares. Il est donc assez logique qu'une partie de sa production reflète l'évolution de l'actualité législative, rende compte des politiques publiques et se saisisse des données recueillies par la statistique publique.

\section{Une tonalité administrative présente aux débuts de la revue}

Dans les premières décennies de la revue, les articles traitant du droit du travail sont ainsi très directement liés à l'action publique en matière de travail et d'emploi. 
Certains sont des synthèses de rapports publics ${ }^{2}$ ou de journées d'études ${ }^{3}$, ou portent sur des réformes contemporaines ou à venir, et ce, au moins jusqu'en $2003^{4}$. D'autres articles présentent des dispositifs étrangers précis, comme le droit de retrait de son poste de travail au Danemark (HINGEL, 1985, $T E \mathrm{n}^{\circ} 25$ ) ou l'expertise en technologie en Allemagne (KESSLER, 1990, TE $\mathrm{n}^{\circ}$ 43). Cette association à l' action publique se manifeste surtout par la prédominance des articles abordant ce qu'on peut appeler un droit de l'emploi (PÉLISSIER, 1999), c'est-à-dire les dispositifs juridiques mis en place par les politiques de l'emploi. Les restructurations, les contrats de travail atypiques, la cessation anticipée d'activité et la réduction du temps de travail sont les principales thématiques présentes dans les 45 articles (soit $2 \%$ du corpus) abordant directement le thème du droit. Ces sujets mis à part, les articles interrogeant la mise en œuvre du droit (sur les 39 heures, le droit d'expression des salariés, les licenciements collectifs) se concentrent dans les deux premières décennies de la revue ; à l'inverse, le thème des discriminations apparaît en 1998 (cf. infra). S'inscrivant dans une tendance plus générale observée au cours des quarante dernières années, Travail et Emploi témoigne ainsi d'un basculement progressif d'un droit pensé à partir de la loi vers une analyse en termes de droits des personnes.

En lien avec l'actualité législative et les évolutions de l'action publique, les politiques de l'emploi constituent, de manière assez logique, une thématique importante de la revue depuis son lancement : si l'on énumère simplement le nombre d'articles qui abordent cette thématique ${ }^{5}$, on voit que cela concerne 128 articles, soit $13 \%$ de ceux publiés en quarante ans d'existence. Sur un thème proche, notons que 135 articles portent sur le chômage, la précarité et/ou la protection sociale. Ces articles témoignent tout d'abord de la difficulté à circonscrire précisément le champ des politiques de l'emploi, puisqu'ils concernent pour certains des politiques macro-économiques et des réformes du marché du travail (baisses de cotisations sociales, réduction du temps de travail, par exemple), tandis que d'autres, la majorité en fait, se concentrent sur des dispositifs visant directement à réduire le chômage (mesures ciblées, etc.). Les politiques de soutien au revenu des personnes sans emploi ou à faibles revenus sont également abordées (revenu minimum d'insertion [RMI], revenu de solidarité active [RSA]), mais de façon secondaire, étant à la frontière entre politiques de l'emploi et politiques sociales. La nécessité de décrire ce vaste ensemble de politiques et de proposer des mesures de leur développement apparaît au travers d'articles de bilan, qui portent notamment sur les dépenses (Colin, 1981, TE n ${ }^{\circ} 8$; Roguet, SALZBERG, 1991, TE nº 48).

2. Tel l'article de Bernard RetTENBACh publié dans le premier numéro de la revue (1979). Intitulé « Diversité des formes juridiques de travail et restructuration des entreprises », cet article propose la synthèse d'un rapport remis au ministère de la Justice.

3. Comme l'article d'Alain SuPIOT sur l'application du droit du travail en Europe, paru en 1991 (TE n ${ }^{\circ} 47$ ), ou la synthèse d'une journée d'études sur les petites et moyennes entreprises (PME) et les relations sociales, parue en 1995 (BARANGER, 1995, TE n ${ }^{\circ}$ 63). N.B. : les références aux articles parus dans Travail et Emploi sont suivies des initiales de la revue et du numéro dans lequel le texte a été publié.

4. Cf. l'article de Marie-Laure Morin, en 2003 ( $T E \mathrm{n}^{\circ}$ 95), sur la réforme de la formation professionnelle continue.

5. Les thèmes (et grands thèmes) dont il est question dans la suite du texte sont ceux de la classification établie et reproduite dans le tableau, mais désormais, il s'agit d'un comptage simple : nous comptons par exemple le nombre d'articles rattachés au thème « politique de l'emploi » dans la liste totale des 980 articles, quel que soit le nombre de thèmes auquel cet article renvoie. 


\section{Un foisonnement de politiques de l'emploi, en partie inspirées d'expériences étrangères}

Dans ce champ des politiques publiques, les publications de Travail et Emploi témoignent de la prégnance des références à la comparaison internationale. Les exemples étrangers font régulièrement l'objet d'approches centrées sur un dispositif (l'aménagement du temps de travail en Belgique [TUCHSZIRER, 1986, TE ${ }^{\circ} 28$ ], l'activation dans les pays scandinaves [Kosonen, 1999, TE $\mathrm{n}^{\circ} 79$ ]) ou de mises en perspective comparées (France-Suède, ERHEL, 1999, $T E \mathrm{n}^{\circ} 79$; Wallonie-France, BERTHET, CONTER, 2011, TE $\mathrm{n}^{\circ}$ 125). Cette orientation n'est d'ailleurs pas réservée aux politiques de l'emploi. En effet, la revue s'inscrit dans le temps long de l'évolution des institutions européennes, de leurs implications législatives et de leurs effets sur le champ du travail et de l'emploi. En témoigne par exemple la série d'articles portant sur le comité d'entreprise européen, de sa création au milieu des années 1990 (HOARAU, JACQUIER, 1995, TE $\mathrm{n}^{\circ}$ 65) jusqu'à la dernière directive adoptée en 2011. Dans cet intervalle, Travail et Emploi se fait l'écho de la place du dispositif dans le fonctionnement de la représentation des salariés au niveau européen, dans le contexte de l'élargissement de l'Union aux pays de l'Est (Bethoux, 2004, TE n ${ }^{\circ} 98$; Delteil, 2006, TE n ${ }^{\circ} 106$; Delteil, Dieuaide, 2010, TE n ${ }^{\circ} 123$ ).

En dynamique, les articles de la revue font émerger plusieurs périodes. La « naissance » des politiques de l'emploi ciblées se fait avec la « rupture » de 1977 (CoLIN et al., 1981, $T E \mathrm{n}^{\circ}$ 10) et les premières mesures jeunes (Pactes nationaux pour l'emploi des jeunes, abordés par Frédérique PATE et ses coauteurs en 1980, TE n ${ }^{\circ}$ 6), initiant une phase de développement de dispositifs diversifiés, souvent éphémères (travaux d'utilité collective, emplois d'initiative locale, stages d'initiation à la vie professionnelle). Les années 1990 voient l'apparition des mesures générales de baisse du coût du travail (HenRiot-Olm, 1997, TE $\mathrm{n}^{\circ} 73$; Assouline et al., 1997, TE $\mathrm{n}^{\circ}$ 73), tandis que l'idée de la réduction du temps de travail comme outil de lutte contre le chômage, déjà avancée en 1980 (BOISARD, 1980, $T E \mathrm{n}^{\circ}$ 3), s'affirme avec les lois Robien puis Aubry (CETTE, Gubian, 1998, TE $\mathrm{n}^{\circ} 74$; BLOCH-London et al., 1999, TE n ${ }^{\circ}$ 79). Depuis le tournant du siècle, les dispositifs abordés sont plus hétérogènes (formation continue, évaluation $e x$ post du RMI puis suivi de la mise en œuvre du RSA, accompagnement des demandeurs d'emploi, emploi à domicile, Garantie jeunes, etc.), reflétant la multiplicité des outils mis en œuvre et le développement des méthodes quantitatives d'évaluation de ces dispositifs.

Cette multiplicité s'accompagne d'un déplacement progressif des politiques de lutte contre le chômage, qui se modifient petit à petit sous l'influence de l'Union européenne et des théories économiques, avec des articles de plus en plus nombreux analysant par exemple le profilage (ou ciblage) des demandeurs d'emploi, les modalités de leur accompagnement, le contrôle de leurs recherches, la durée de leur indemnisation ou l'externalisation de leur suivi à des opérateurs privés de placement. L'objectif de retour à l'emploi, et plus largement d'augmentation du taux d'emploi, dans une 
perspective d'activation et de New Public Management concerne toutes les franges de la population : les femmes comme les hommes, les jeunes comme les plus âgés. On mesure ainsi bien l'évolution des politiques conduites par rapport au début de la période, où les articles s'intéressaient aux cessations anticipées d'activité comme instruments de lutte contre le chômage. De fait, si le vieillissement des actifs est un thème abordé avec une grande régularité par la revue depuis ses premières années, son traitement s'est profondément modifié : l'analyse du déploiement des régimes de préretraite a rapidement laissé place à leur remise en cause puis, à partir des années 2000, à la promotion législative des enjeux de maintien dans l'emploi des salariés âgés.

Il en va de même concernant le traitement social du chômage. À partir des années 2000, la précarité et le chômage de longue durée ne sont plus uniquement abordés, comme ils l'étaient dans les années 1980 ou 1990, sous l'angle de la pauvreté (ou du « sous-prolétariat », cf. DeCOUfLÉ, 1983, TE n ${ }^{\circ} 16$; GODINOT, JoIN-LAMBERT, 1984, $T E \mathrm{n}^{\circ} 21$ ), de la montée de la thématique de l'exclusion et de l'instauration du RMI. Les décennies suivantes, ce sont les notions de sécurisation des transitions, de marché transitionnel et de flexicurité qui s'imposent, par exemple avec le numéro consacré à cette question en 2008 (cf. entre autres GAZIER, 2008 et LEFEBVRE, MÉDA, 2008, $\left.T E \mathrm{n}^{\circ} 113\right)$. L'année suivante, le numéro consacré à la stratégie de l'Organisation de coopération et de développement économiques (OCDE) pour l'emploi est l'occasion de revenir sur la tension existant entre sécurité et flexibilité (FREYSSINET, 2009, TE $\mathrm{n}^{0} 118$ ) en plus de présenter les positions des économistes néoclassiques en matière de lutte contre le chômage (cf. CAHUC, 2009 et LAROQUe, 2009, TE n ${ }^{\circ} 118$ ). C'est dans ce contexte qu'apparaît le RSA, nouvel outil, emblématique de la période, dont l'appropriation par les acteurs et les conséquences sur les trajectoires de bénéficiaires sont analysées. Le champ des politiques de l'emploi apparaît comme un domaine de controverses politiques et de débats scientifiques sur le choix des meilleurs outils dans la lutte contre le chômage, de l'article de Günther SCHMID sur le plein-emploi en 1995 $\left(T E \mathrm{n}^{\circ} 65\right)$ au numéro sur les institutions du travail en 2015 (TRAVAIL ET EMPLOI n ${ }^{\circ} 142$ ).

\section{Des débats scientifiques et des controverses politiques, l'exemple de la réduction du temps de travail}

Dans l'ensemble, les publications de la revue traduisent bien à la fois l'empilement des dispositifs et des logiques d'intervention qui caractérise les politiques de l'emploi françaises, ainsi que les incertitudes fortes sur l'efficacité des mesures successivement mises en place, et la place croissante qu'y prennent, parfois de façon controversée, les outils inspirés d'expériences étrangères ou d'incitations européennes. Dans ce cadre, la réduction du temps de travail peut être considérée comme une originalité française. La thématique occupe une place de choix dans les pages de Travail et Emploi dans les années 1990 et du début de la décennie 2000 : elle est envisagée au travers d'approches et méthodes variées, monographies d'entreprises (PÉPIN, TONNEAU, 1979, $T E \mathrm{n}^{\circ} 1$; LABIT, ThOEMmES, 1995, $T E \mathrm{n}^{\circ}$ 64), exemples étrangers (TuChSZIRER, 1986, 
$T E \mathrm{n}^{\circ} 28$; Garonna, Reboani, 1991, $T E \mathrm{n}^{\circ} 48$ ), récits historiques (SouamaA, 2007, $T E \mathrm{n}^{\circ} 110$ ), mais aussi débats théoriques (par exemple autour de l'article de CAHUC, GRANIER, 1998, $T E \mathrm{n}^{\circ}$ 74) ou analyses empiriques. Ainsi, les évaluations des dispositifs successivement mis en place en France (39 heures en 1982, puis 35 heures entre 1998 et 2000) sont très présentes dans la revue, notamment au milieu des années 1980 et entre 1998 et 2002 (cf. par exemple GuBIAN, 2000, TE n 83 ; PÉlisSE, 2000, $T E \mathrm{n}^{\circ} 82$ ) : il s'agit plutôt d'analyses du suivi et de la mise en œuvre des réformes, incluant des exemples d'accords, d'analyses centrées sur des enjeux organisationnels en entreprise, plutôt que d'évaluations d'impact ; pour les 35 heures, de telles évaluations ont été publiées dans Économie et Statistique ${ }^{6}$, ce qui traduit le positionnement de la revue qui se situe à la frontière de l' analyse économique et d'analyses sociologiques interrogeant l'hétérogénéité des pratiques et les effets pour différents groupes sociaux.

La présence de la thématique de la durée du travail a nettement décru depuis le milieu des années 2000, témoignant d'un moindre intérêt politique comme académique pour ce sujet sur la période récente. De plus, on relève une transformation des questions alors abordées en matière de durée du travail, qui s'éloignent de la problématique de la réduction du temps de travail comme dispositif de création d'emplois. La thématique du temps partiel, présente dès les années 1980 (KERGOAT, 1984, TE n ${ }^{\circ} 21$ ), demeure régulièrement étudiée (BUÉ, RouX-Rossi, 2002, TE n 90 ; BUÉ et al., 2013, TE n ${ }^{\circ} 134$ ). Plus récemment, on voit s'affirmer des questions portant sur la soutenabilité et la pénibilité des horaires, par exemple des horaires atypiques, notamment dans certaines professions ou secteurs : dans la pêche (CHARVET et al., 2016, TE n ${ }^{\circ} 147$ ), à l'hôpital (BENALlaH, Domin, 2017, TE ${ }^{\circ}$ 152) ou dans les grands magasins (Grimaud, 2018, $\left.T E \mathrm{n}^{\circ} 155-156\right)$.

\section{De la lutte contre les inégalités à l'analyse des discriminations}

L'évolution des politiques publiques est également visible dans un champ qui déborde les seules questions liées au travail et à l'emploi, celui de la lutte contre les inégalités. En effet, si cette thématique est présente dès les débuts de la revue - qui, dans son deuxième numéro, publie un article dédié à l'évolution des inégalités au travail et sur le marché de l'emploi (TReutenAere, 1979, $T E \mathrm{n}^{\circ} 2$ ) - elle ne fait pas l'objet des mêmes approches au fil des quarante ans de la revue.

Durant les vingt premières années, elle est d'abord appréhendée à l'aune de catégories de travailleurs et travailleuses : femmes en tout premier lieu, mais aussi jeunes - et plus tardivement travailleurs âgés - et, dans une moindre mesure, étrangers ou immigrés (les deux étant tenus alors pour synonymes ${ }^{7}$, et ce, jusqu'en 1990, où le recensement de la population distingue pour la première fois les personnes de nationalité étrangère de celles nées étrangères à l'étranger et ayant donc pu acquérir

6. Cf. son numéro spécial paru en 2004 (n³ 376-377).

7. Cf. notamment Villey, 1980, TE n 4 ; MAYere, 1983, $T E \mathrm{n}^{\circ} 17$. 
ultérieurement la nationalité française), catégories qui sont ainsi inégalement étudiées. Si l'emploi et le travail (domestique et salarié) des femmes (KeRGOAT, 1984, $T E \mathrm{n}^{\circ} 21$ ), puis plus largement les inégalités de genre dans la vie professionnelle - ou dans la (difficile) conciliation vie professionnelle/vie familiale (GARNER et al., 2005, $T E \mathrm{n}^{\circ} 102$; TORNs, 2008, TE $\mathrm{n}^{\circ} 115$ ) - et les statuts d'emploi analysés sous un angle genré (TRAVAIL ET EMPLOI n ${ }^{\circ} 150,2017$ ) font assez régulièrement l'objet d'articles au fil des quarante ans de la revue, il n'en va pas de même des travailleurs étrangers et/ou immigrés ${ }^{8}$. La montée en puissance des politiques d'égalité dans le champ du travail et de l'emploi, envisagées d'abord dans une perspective d'égalité hommes-femmes, d'un côté, et la suspension de l'immigration de main-d'œuvre à compter de juillet 1974, la déconnexion progressive des politiques d'emploi et d'immigration à compter de la fin des années 1970, et la tutelle du ministère de l'Intérieur sur les questions d'immigration ces quarante dernières années, de l'autre, expliquent sans nul doute ce traitement différencié.

En parallèle de ces entrées par catégories de population, la revue publie plus ponctuellement des articles généraux, voire des dossiers sur l'égalité professionnelle - à l'instar de celui consacré aux " politiques d'égalité professionnelle en entreprise », abordée principalement sous l'angle du genre et, dans une moindre mesure, de la génération (TRAVAIL ET EMPLOI $\left.\mathrm{n}^{\circ} 102,2005\right)$ - ou, plus tardivement, sur les discriminations. Là encore, on mesure les effets de cadrage de l'action publique : les premiers textes traitant des différenciations entre catégories de travailleurs et travailleuses sous l'angle des discriminations ${ }^{9}$ paraissent à compter de 1997, dans la période de mise à l'agenda européen, puis français, de la lutte contre les discriminations, et surtout des directives européennes en matière de discriminations fondées sur le sexe (Directive 97/80/CE du Conseil du 15 décembre 1997) et la race ou l'origine ethnique (Directive 2000/43/ CE du 29 juin 2000). C'est avant tout sous l'angle des enjeux et avancées juridiques que les articles parus dans la revue analysent les discriminations (MiNÉ, 1999, TE $\mathrm{n}^{\circ} 80$; BouMAHDI et al., 2005, TE $\mathrm{n}^{\mathrm{o}} 104$ ), plus tardivement sous celui des pratiques et perceptions de la discrimination, en l'occurrence syndicale (JEFFERYS, 2016, TE $\mathrm{n}^{\circ} 146$; GIRAUD et al., 2016, TE $\mathrm{n}^{\circ} 145$ ).

\section{Une ressource : la richesse des enquêtes statistiques françaises sur le travail et l'emploi}

En plus du droit et de l'action publique, l'élaboration de sources statistiques originales a également contribué à façonner la production de la revue. Travail et Emploi a en effet accompagné la création de nouvelles enquêtes statistiques, dont la richesse est particulièrement importante en France dans ce domaine (GOLLAC, VOLKOFF, 2010).

\footnotetext{
8. Avec sept articles, il s'agit de la catégorie de population la moins traitée au fil des quarante ans de la revue, parmi celles précitées.

9. La discrimination étant entendue comme une « différence de traitement illégitime : illégitime parce qu'arbitraire, et interdite puisqu'illégitime », selon la définition devenue de référence qu'en a donnée Danièle LOCHAK (1987, p. 778).
} 
C'est ainsi que le thème des conditions de travail est présent dès les premiers numéros de la revue, de 1980 à 1982, se faisant l'écho des résultats de la première enquête Conditions de travail (1978). En 1996, un numéro spécial ( $n^{\circ}$ 66) est édité autour de la première édition de l'enquête Relations professionnelles et négociations d'entreprise (REPONSE). Dans la décennie qui suit, un volume important d'articles s'intéresse aux instances de représentation du personnel et aux relations sociales en entreprise en s'appuyant sur les résultats des deux premières éditions de l'enquête (1993 et $1998^{10}$ ). Notons toutefois que, dans un cas comme dans l'autre, une fois l'enquête installée dans le paysage de la production statistique, la revue publie nettement moins de travaux qui la mobilisent ${ }^{11}$. Et certaines enquêtes statistiques apparues au cours des quarante dernières années n'ont pas fait l'objet d'une valorisation spécifique par la revue : c'est le cas de l'enquête Surveillance médicale des expositions des salariés aux risques professionnels (Sumer : 1994 ; 2003 ; 2010 ; 2017), de l'enquête Santé et itinéraire professionnel (SIP : 2006-2010) ou encore de l'enquête Offre d'emploi et recrutement (Ofer : 2005).

\section{Une évolution du regard porté par les sciences sociales sur le travail et l'emploi}

Parmi les thématiques émergentes dans la revue, certaines s'appuient non seulement sur de nouvelles enquêtes statistiques mais témoignent aussi de nouveaux questionnements.

\section{De l'organisation du travail à la gouvernance des entreprises}

L'organisation du travail, qui a particulièrement été traitée dans les décennies 1990-2000, principalement sous l'angle des transformations technologiques, en est un exemple. Après trois premiers articles sur l'informatisation dans les années 1980, plusieurs articles présentent les résultats d'une première enquête sur la technique et l'organisation du travail (menée en 1987), préfigurant l'enquête Changements organisationnels et informatisation (COI). Une autre façon d'aborder l'organisation du travail consiste à identifier des « modèles » organisationnels, pour notamment interroger la place qu'ils ménagent à la participation des salariés. On trouve ainsi ces questions d'évolution des modèles de production dans des articles sur le taylorisme, qui posent non seulement la question de l'organisation du travail (PASTRÉ, 1983, $T E \mathrm{n}^{\circ} 18$ ), mais aussi celle de l'intensité du travail avec la diffusion des contraintes marchandes au

10. L'enquête de 1998 donne lieu notamment à des articles publiés dans deux numéros de l'année $2003\left(\mathrm{n}^{\circ} 93\right.$ et $\left.\mathrm{n}^{\circ} 95\right)$. 11. Dans le cas de l'enquête REPONSE, un ouvrage collectif paru à La Découverte (AMOSSÉ et al., 2008) a remplacé les dossiers de la revue pour l'édition de 2004. L'enquête REPONSE de 2010 n'a quant à elle donné lieu qu'à deux articles de la revue sur la discrimination syndicale (en 2016). 
secteur industriel (CÉZARD et al., 1992, $T E \mathrm{n}^{\circ}$ 54). Étonnamment - y compris au cours de la période récente (et alors que de nombreuses enquêtes ont travaillé la question d'un néotaylorisme dans le secteur tertiaire) - peu d'articles portent sur l'organisation des entreprises de services (BUSCATTO, 2001, TE $\mathrm{n}^{\circ}$ 87) comme si le terme «entreprise » était nécessairement attaché à l'industrie, les services étant davantage étudiés sous l'angle des professions ou des conditions de travail. Les questions de «nouvelles technologies ", d'innovation et de rationalisation sont régulièrement traitées au cours de ces deux décennies, en lien avec l'adaptation des travailleurs et des emplois (COUTROT, 1995, $T E \mathrm{n}^{\circ}$ 64) et la formation professionnelle (HARDY-DUBERNET, 1998, $\left.T E \mathrm{n}^{\circ} 77\right)$.

Les questions organisationnelles disparaissent quasiment de la revue dans les années 2010 pour laisser la place à des analyses sur les modes de gouvernance des entreprises, qui apparaissent quant à elles dans les années 2000 (DEAKIN, 2007, TE $\mathrm{n}^{\circ} 109$; Lefresne, SAuviat, 2009, TE $\mathrm{n}^{\circ}$ 117). L'article de Denis Segrestin en 1990 $\left(T E \mathrm{n}^{\circ} 46\right)$ est l'un des derniers à traiter de manière frontale la question de l'entreprise en sociologie. L'auteur constate que celle-ci est devenue une « catégorie pertinente de la sociologie »-grâce notamment aux travaux de l'économie des conventions mais s'interroge sur le paradoxe suivant : « les paradigmes à partir desquels s'opère aujourd'hui le renouveau de la représentation de l'entreprise n'ont[-ils] pas pour propriété de ne dessiner qu'une théorie de la diversité des entreprises, incapable de ce fait de nous renseigner sur ce qui se joue réellement du point de vue de l'évolution de la société » (p. 28). Les titres et les mots-clés sont particulièrement évocateurs de l'évolution des études et des concepts dans les travaux de sociologie, de sciences politiques ou de gestion, mais aussi plus généralement dans la manière d'envisager l'exercice du pouvoir dans les sphères politiques et économiques : le passage de la «gestion » au « gouvernement » puis à la « gouvernance » est ainsi le signe de la volonté d'étudier tous les niveaux d'exercice du pouvoir, le terme «gouvernance » ayant toutefois tendance - lorsqu'il n'est pas l'objet d'une analyse réflexive - à euphémiser l'inégale répartition du pouvoir (quelques articles font référence à la gestion, principalement pour aborder la question de la main-d'œuvre et des ressources humaines). Dans son article consacré à la question de la participation des salariés face à la montée en puissance des marchés financiers, Antoine REBÉRIOUX (2003, TE $\mathrm{n}^{\circ}$ 93) indique par exemple que le thème de la gouvernance d'entreprise a pris une importance croissante dans les débats sur les transformations du secteur productif pour désigner « l'ensemble des dispositions permettant aux actionnaires de s'assurer un retour maximal sur leur investissement » en dépit de l'apparente place accordée aux salariés dans la gestion des entreprises (p. 25). Il insiste ce faisant sur le développement d'une définition particulière de l'entreprise - devenue « firme »-qui est intimement liée au développement du paradigme de la «valeur actionnariale » (shareholder value) (FLIGSTEIN, 1990), selon lequel l'intérêt des actionnaires devient le principal, si ce n'est l'unique, objectif de l'entreprise. 


\section{Le déclin des approches macro-économiques, la montée de l'évaluation micro-économétrique}

Au cours de ces quatre décennies, l'approche macro-économique des questions de chômage et d'emploi s'est progressivement effacée au profit de travaux de nature micro-économique. On retrouve pour partie cette évolution inhérente à l'ensemble de la discipline lorsqu'on analyse les articles publiés dans Travail et Emploi. Au début des années 1980, les articles traitent en effet des phénomènes économiques sous l'angle de la planification et, toujours au niveau macro, des liens entre coût du travail et emploi, ou entre durée du travail et emploi. Par la suite, la question de la planification disparaît de la revue avec l'extinction de ce mode de régulation de l'économie et de la société. Emploi et chômage sont alors abordés sous l'angle du fonctionnement du marché du travail seul, en lien avec les problèmes d'appariement liés aux caractéristiques individuelles, à l'(in)efficacité des institutions et politiques d'emploi mises en œuvre pour lutter contre le chômage, laissant en arrière-plan, comme élément de contexte, l'environnement macro-économique. L'approche macro-économique ressurgit néanmoins au fil des crises. Ainsi, au cours des années 1990, face à un environnement conjoncturel dégradé et un haut niveau de chômage, le débat porte sur les conditions nécessaires pour qu'une réduction du temps de travail soit favorable à l'emploi, et la revue publie plusieurs articles sur l'évaluation macro-économique de la baisse du temps de travail. Puis, au moment de la récession de 2009, la revue publie un numéro sur la stratégie de l'OCDE pour l'emploi, qui remet sur le devant de la scène le rôle des politiques macro-économiques pour favoriser la croissance et lutter contre le chômage (TRAVAIL ET EMPLOI n ${ }^{\circ} 118,2009$ ). Dans ces contributions, les questions autour de la durée du travail laissent la place à celles portant sur les politiques de soutien à la demande, leur efficacité et leur articulation avec les réformes structurelles et les aspects réglementaires du marché du travail.

Les quatre décennies de Travail et Emploi donnent également à voir la montée des questions autour de l'évaluation des politiques économiques. À partir des années 2000, la question de l'évaluation des dispositifs et de leur impact estimé devient centrale dans les articles, que ces dispositifs soient français ou étrangers, tels que le New Deal for Young People (DAvoine, 2005, TE $\mathrm{n}^{\circ} 101$ ) ou encore des mesures allemandes (Stephan, 2014, TE $\mathrm{n}^{\circ} 139$; AMABLE, Françon, 2015, TE $\mathrm{n}^{\circ}$ 142). Par ailleurs, alors que l'évaluation s'affirme dans le champ des politiques de l'emploi, des publications sont directement consacrées aux questions méthodologiques qu'elle pose, avec notamment un débat sur l'expérimentation en 2013 (Gomel, SERVERIN, 2013, $T E \mathrm{n}^{\circ} 135$; BUREAU et al., 2013, TE $\mathrm{n}^{\circ}$ 135) et un numéro spécial sur l'évaluation des politiques actives du marché du travail en 2014 (TRAVAIL ET EMPLOI n ${ }^{\circ} 139,2014$ ). Plus largement, de nouvelles formes d'évaluation des politiques de l'emploi se développent dans les pages de la revue : aux travaux explicitant leurs enjeux par l'analyse de leur genèse, proposant des estimations ex ante de leurs effets ou restituant leur réception sur le terrain viennent s'ajouter des évaluations quantitatives ex post, à visée causale, qui entendent mesurer leurs effets par des techniques statistiques complexes (FERRACCI, 
Martin, 2013, $T E \mathrm{n}^{\circ} 135$; Fremigacci, Terracol, 2014, TE $\mathrm{n}^{\circ} 139$; Moczall, 2014, $T E \mathrm{n}^{\circ} 139$ ). Pour autant, la revue ne renonce jamais à publier des travaux de différentes natures, comme l'illustre le numéro récent sur la Garantie jeunes, où sont présentées une comparaison internationale, une évaluation quantitative et deux enquêtes de terrain auprès des acteurs locaux et des bénéficiaires (TRAVAIL ET EMPLOI n ${ }^{\circ} 153,2018$ ).

\section{Le développement de la sociologie du chômage et des professions}

Si le déclin des approches macro-structurelles et l'émergence des travaux centrés sur les situations individuelles sont particulièrement visibles en économie, des évolutions parallèles peuvent être observées en sociologie, et notamment en sociologie de l'emploi. Ainsi, au tournant des années 2000, plusieurs articles témoignent d'une socioéconomie du chômage, de l'emploi et du marché du travail en plein développement, qui décrit au travers d'observations et de récits situés l'expérience des demandeurs d'emploi, le travail des acteurs locaux de l'emploi (missions locales et agences de l'ANPE) et les pratiques de recrutement (cf. par exemple Delfini, DEMAZIÈre, 2000, TE $\mathrm{n}^{\circ} 81$; DivAY, 2000, TE $\mathrm{n}^{\circ} 81$ ).

Ce glissement se retrouve également dans des articles présentant des monographies analysant de près le contenu d'un métier, les conditions de travail d'une activité ou le devenir d'un groupe professionnel. Travail et Emploi apparaît ainsi à juste titre comme un support privilégié pour proposer des zooms sur tel ou tel métier ou pour réfléchir aux questions de trajectoires professionnelles. Pour autant, l'accélération des publications sur les professions à partir du début des années 2010 peut sans doute s'expliquer par deux éléments qui dépassent le positionnement éditorial de Travail et Emploi mais que la revue permet de saisir si on regarde l'évolution de ses sommaires. Le premier élément est l'actualité scientifique du tournant des années 2010 dans le sous-champ de la sociologie des professions, en particulier en France, qui s'est traduit par une multiplication des thèses, et plus généralement des recherches, sur les groupes professionnels suivant des approches méthodologiques et théoriques renouvelées : les ouvrages de Didier Demazière et Charles GAdÉA (2009), de Valérie BousSARD, Didier DemaZière et Philippe Milburn (2010) ou encore celui de Florent ChAMPY (2012) en proposent des synthèses. Le second élément, plus structurant que le précédent, est celui de l'arrivée massive des interrogations sur les effets du genre et sur les rapports sociaux de sexe dans les travaux de recherche français. Avec des articles portant sur les situations de travail des femmes, notamment des ouvrières, la question du genre était bien sûr déjà présente dès les années 1980 et 1990, mais elle s'est nettement renforcée au cours des dix dernières années. Beaucoup d'articles (douze depuis 2011) s'intéressent ainsi à ce que le genre fait aux carrières ou aux groupes professionnels en proposant un questionnement plus général sur les rapports sociaux de sexe ou sur les inégalités entre les femmes et les hommes ${ }^{12}$. Si ces interrogations se sont développées au-delà

12. Par exemple, les articles de Isabel Boni-LE GoFF « "Ni un homme, ni une femme mais un consultant”. Régimes de genre dans l'espace du conseil en management » (2012, TE $\mathrm{n}^{\circ}$ 132) ou de Arnaud DuPRAY et Stéphanie MouLLET, 
des recherches sur les professions et le travail, les analyses des univers professionnels et des inégalités au travail et face à l'emploi permettent de saisir les problématiques liées au genre de manière privilégiée.

\section{Les transformations du champ des relations professionnelles}

Le champ des relations de travail et du syndicalisme est l'un des plus abondamment traités dans la revue, et ce, de façon relativement constante, avec plus d'une quarantaine d'articles parus au cours de chaque décennie (soit 199, autrement dit $20 \%$ du corpus total). Dans ce champ, la négociation collective occupe une place particulièrement importante (près d'un tiers des articles) ; les organisations syndicales et, très secondairement, patronales font l'objet d'une quarantaine d'articles. Par contraste, on recense quatre fois moins d'articles portant sur les conflits du travail que sur la négociation collective, et le thème de la participation des travailleurs reste marginal et concentré dans les numéros du milieu des années 1980 (douze articles au total, dont trois publiés au cours de la première décennie). La façon de traiter chacune de ces thématiques dans la revue a fortement changé depuis 1979, reflétant assez bien l'évolution plus générale des études sur les relations professionnelles.

Dans les premières années de la revue, les articles s'intéressant aux relations de travail le font souvent à partir des politiques du travail et de l'emploi alors mises en œuvre. Tel est le cas du texte sur les comités de bassin d'emploi (MicheAu, 1982, TE $\mathrm{n}^{\circ} 11$ ) ou de celui sur le salaire minimum interprofessionnel de croissance (Smic ; BuGHIN, 1982, TE $\mathrm{n}^{\circ}$ 14). La négociation collective est principalement appréhendée comme une négociation salariale, le plus souvent à l'échelle de la branche. C'est à cette période que la revue fait connaître les travaux qui se développent alors sur les grilles de classification conventionnelles (cf. par exemple : SAGLIO, 1986, $\left.T E \mathrm{n}^{\circ} 27\right)$. Le thème de l'expression et de la participation des salariés est également présent, en écho aux nouveaux droits instaurés par les lois Auroux en 1982, en particulier dans le cadre du numéro 24 (1985). La conflictualité du travail est abordée sous deux angles : soit les articles développent une analyse statistique des grèves (DASSA, 1981, TE $\mathrm{n}^{\circ} 7$ pour l'année 1976 ; Furjot, Feuerbach, 1986, TE n ${ }^{\circ} 28$ pour 1985 ; FurJot, Noel, 1987, $T E \mathrm{n}^{\circ} 34$ pour 1986), soit ils rendent compte de la forte conflictualité sociale en GrandeBretagne durant l'ère Thatcher (BURGI, 1986, TE n 30 ; BRIDGFORD, STIRLING, 1987, $\left.T E \mathrm{n}^{\circ} 34\right)$. En matière de relations professionnelles, les cas étrangers sont d'ailleurs assez présents dans les colonnes de la revue, surtout pour présenter les syndicalismes étrangers (au Japon, aux États-Unis et dans les pays d'Europe occidentale). Par contre, le syndicalisme français est peu abordé, si ce n'est pour traiter des audiences syndicales (AujARD, VolKOFF, 1986, $T E \mathrm{n}^{\circ}$ 30) ou de la réforme des prud'hommes de 1979 (BONAFE-SCHMITT, 1983, TE n ${ }^{\circ} 18$ ).

«Le salaire moindre des femmes : une question d'individus ou de profession ?» $\left(2015, T E \mathrm{n}^{\circ} 144\right)$, ou encore de Régine BERCOT, « Le devenir des femmes en chirurgie : la diversité des mondes de socialisation » $\left(2015, T E \mathrm{n}^{\circ} 141\right)$. 
Cette tendance à présenter des syndicalismes étrangers se prolonge dans les années 1990 alors que des premiers travaux de politistes sur les syndicats français sont également publiés (LABBÉ, 1994, TE n ${ }^{0} 59$; BeVORT, 1995, TE nº 62 ; ANDOLFATTO, 1997, $\left.T E \mathrm{n}^{\circ} 71\right)$. Notons également en 1997 un premier article sur les organisations patronales, rédigé par Jean BUNEL ( $T E \mathrm{n}^{\circ} 70$ ). On ne dénombre que deux articles traitant des conflits du travail entre 1989 et 1998 ; les articles sur la négociation collective dominent, puisqu'ils représentent près de la moitié des articles étudiant les relations de travail et le syndicalisme. Si les salaires restent un enjeu des relations entre partenaires sociaux, ils sont assez peu présents dans les pages de la revue. Et on voit apparaître de « nouveaux » thèmes de négociation, comme le temps de travail (BosCH, LaLlement, 1991, TE $\mathrm{n}^{\circ} 49$ ), l'intéressement (COUTROT, 1992, TE $\mathrm{n}^{\circ} 53$ ) et l'emploi (BARALDI, LAMOTTE, 1998, TE $\mathrm{n}^{\circ}$ 76). Ce qui marque le plus cette décennie, c'est le développement des articles portant sur les relations sociales en entreprise, en écho à l'enquête REPONSE dont la première édition remonte à la même période (cf. supra).

Entre 1999 et 2008, les proportions d'articles consacrés à chacune des grandes thématiques du champ restent relativement stables. Quelques déplacements sont toutefois perceptibles. Plusieurs articles analysent les positions et doctrines syndicales (cf. par exemple : BRUCY, 2001, TE $\mathrm{n}^{\circ} 86$; GRIMAULT, 2008, TE $\mathrm{n}^{\circ} 113$ ), un autre les trajectoires des anciens militants syndicaux (UBBIALI, 1999, $T E \mathrm{n}^{\circ}$ 80). Paraissent également des articles étudiant les politiques sociales des entreprises, sans que les catégories classiques des relations professionnelles (syndicats, représentation des salariés, négociation, conflit) ne soient mobilisées par leurs auteurs pour les analyser : la responsabilité sociale des entreprises (BONVIN, BADAN, 2007, $T E \mathrm{n}^{\circ} 109$ ), les politiques salariales des entreprises (BARREAU, BROCHARD, 2003, $T E \mathrm{n}^{\circ}$ 93), l'influence des marchés financiers sur les politiques sociales d'entreprise (REBÉRIOUX, 2003, TE $\mathrm{n}^{\circ} 93$ ), ou encore l'individualisation des relations salariales avec le développement de la logique «compétence » (ZimMERMANN, 2000,TE $\mathrm{n}^{\circ} 84$ ) sont les thèmes qui $\mathrm{y}$ sont abordés.

La dernière décennie marque une rupture importante. Les articles portant sur la négociation collective sont en très net recul. Ils laissent place à un plus grand nombre d'articles sur les organisations collectives et, dans une moindre mesure, les instances de représentation du personnel et les conflits du travail. Les orientations données par le comité de rédaction dans le cadre de la programmation de numéros spéciaux sont pour beaucoup dans cette rupture. Quatre dossiers (dont deux ayant donné lieu à des numéros doubles) rassemblent près des trois quarts des articles traitant des relations de travail et du syndicalisme au cours des années 2010 : ils portent sur les filiales de multinationales en Europe de l'Est $\left(\mathrm{n}^{\circ} 123,2010\right)$, la représentativité syndicale et patronale $\left(\mathrm{n}^{\circ} 131,2012\right)$, les restructurations en temps de crise $\left(\mathrm{n}^{\text {os }} 137\right.$ et 138,2014$)$ et la discrimination syndicale $\left(\mathrm{n}^{\text {os }} 145\right.$ et 146,2016$)$. 


\section{Un miroir des transformations du travail et de l'emploi}

Il est parfois compliqué de distinguer ce qui relève de l'évolution des champs disciplinaires et ce qui est à relier aux transformations réelles du travail. C'est notamment le cas pour les questions liées à la santé au travail. La revue a ainsi servi de caisse de résonance à la montée en puissance des problématiques de santé psychique, et ce, de façon relativement précoce. Après un article sur le burnout en 2003, un numéro aborde l'année suivante les violences au travail, le harcèlement et le mal-être. Dans les numéros qui suivent, on peut trouver des réflexions sur les outils de gestion, l'évaluation du travail et leurs effets sur les conditions de travail. L'expression « risques psychosociaux » fait elle-même son apparition en 2007 dans un article consacré à l'Espagne (GRAU PINEDA, 2007, TE n ${ }^{\circ}$ 112). Et la notion donne lieu à un numéro spécial pluridisciplinaire présentant, en 2012, un état des lieux des connaissances établies en histoire, sciences de gestion, épidémiologie, psychologie et sociologie (TRAVAIL ET EMPLOI $\mathrm{n}^{\circ} 129$ ). Le succès de la notion ne signifie pas que les travaux plus classiques sur les conditions de travail et la pénibilité aient disparu de Travail et Emploi, comme en témoigne le numéro coordonné en 2016 par Christelle Avril et Pascal Marichalar et intitulé «Quand la pénibilité du travail s'invite à la maison » (TRAVAIL ET EMPLOI $\mathrm{n}^{\circ}$ 147). En débordant la scène du travail, comme les ethnographes ont l'habitude de le faire, les articles montrent l'interaction croissante entre ce qui se déroule dans la sphère professionnelle et ce qui se passe dans l'univers domestique, et permettent de comprendre les enjeux toujours plus importants des liens entre santé et travail.

\section{L'individualisation de la relation salariale}

Pour d'autres objets, il est possible de lire au travers des quarante ans d'articles publiés dans la revue un certain nombre d'évolutions qui ont profondément transformé le monde du travail. C'est notamment le cas de l'individualisation progressive de la relation salariale. Les articles reflètent en effet d'assez près les changements qui affectent sur la période la «gestion du personnel » (BROUDIC, ESPINASSE, 1980, $T E \mathrm{n}^{\circ} 6$ ), voire la « gestion de la force de travail » (FREYSSINET, 1980, $T E \mathrm{n}^{\circ} 4$ ), puis la « gestion de l'emploi » et enfin celle des « ressources humaines». Les premiers articles témoignent de ce qui sera qualifié d' «effritement» de la logique de marché interne, avec la multiplication des licenciements, les recours à la sous-traitance et l'essor des « formes particulières d'emploi », que décrivent dès le début de la décennie 1980 Patrick BROUDIC et Jean-Michel ESPINASSE (1980, TE $\mathrm{n}^{\circ}$ 6), la revue consacrant un colloque international en 1988 et un numéro spécial l'année suivante à ces «nouvelles formes d'emploi » (TRAVAIL ET EMPLOI n ${ }^{\circ} 39,1989$ ). Ces articles font état de la montée en puissance de l'individualisation de la relation salariale, dans les rémunérations, les carrières, au détriment de la logique collective, comme l'illustre l'évolution du vocabulaire : au fil du temps, il est moins question de licenciements et davantage de « gestion des sureffectifs » et de « départs volontaires », reflets des pratiques et des construits sociaux au sein des entreprises (MALLET, 1989, TE ${ }^{\circ} 40$; BEAUJOLIN, 1998, TE $\mathrm{n}^{\circ} 75$ ). 
Le passage de la notion de qualification à celle de compétences (TRAVAIL ET EMPLOI $\mathrm{n}^{\circ}$ 57-2, 1993 ; ZiMMERMANN, 2000, $T E \mathrm{n}^{\circ}$ 84) et l'analyse des dispositifs afférents dans la gestion des carrières (BARRAGAN, HARDY-DuBERNET, 2005, TE $\mathrm{n}^{\circ} 103$ ) soulignent la congruence entre l'individualisation des pratiques en interne et la politique de flexibilité/sécurisation des parcours qui met notamment l'accent sur l'employabilité. Dans les décennies 2000 et 2010, on observe ainsi un nombre croissant d'articles portant sur la sélection à l'embauche et le rôle des entreprises et des intermédiaires dans la construction des mobilités et des transitions individuelles. C'est aussi à partir de la décennie 2000 que les travaux renouent avec une entrée sectorielle des ressources humaines, quelque peu effacée depuis la décennie 1980, à l'image de l'article de Sylvie Monchatre (2018, TE $\mathrm{n}^{\circ}$ 155-156) qui analyse le recrutement sur des emplois peu qualifiés dans la restauration.

\section{Une insertion professionnelle plus difficile, des carrières davantage hachées et toujours inégalitaires}

L'individualisation de la relation de travail peut également se lire en filigrane lorsque l'on considère les articles portant sur l'analyse des mobilités et des trajectoires. Ces questions sont en effet relativement peu présentes dans la revue avant le début des années 1990. Au cours de ces quarante ans, 95 articles se voient attribuer au moins un mot-clé renvoyant à ces thématiques : seuls 15 sont publiés avant 1990. Ces articles des années 1980 sont par ailleurs très marqués par la question du chômage, en particulier par l'explosion du chômage des jeunes dès le milieu des années 1970, et plus généralement par l'entrée dans l'ère du chômage de masse (CHAUvel, 1998 ; PeugnY, VAn DE Velde, 2013). Ils portent sur deux sous-populations spécifiques : les femmes et les jeunes, dont est questionnée la qualité de l'insertion professionnelle, qu'il s'agisse des jeunes ayant effectué un stage en entreprise (SIMON, GouRMELEN, 1979, $T E \mathrm{n}^{\circ} 1$ ), des jeunes stagiaires de l'Association nationale pour la formation professionnelle des adultes (Afpa ; GRISEZ, 1982, TE $\mathrm{n}^{\circ}$ 14) ou des femmes de plus de 30 ans (GONTIER et al., 1981, TE ${ }^{\circ}$ 9).

L'intérêt porté à la question de l'insertion professionnelle des jeunes ne se dément ensuite pas, au moins jusqu'à la fin de la décennie 2000. En 1995, Valérie BEAUDOUIN et Anne-Lise AucouturiER publient ainsi un article reposant sur l'analyse lexicale de plus de 500 parcours de jeunes $\left(T E \mathrm{n}^{\circ} 65\right)$; l'année suivante, Didier DEMAZIÈRE et Claude Dubar proposent eux aussi des récits d'insertion de jeunes peu diplômés (TE $\left.\mathrm{n}^{\circ} 69\right)$ et Éric VERDIER questionne « l'insertion des jeunes à la française » $\left(T E \mathrm{n}^{\circ} 69\right)$; en 1999, Stéphane BEAUD rend compte d' « un cas de sauvetage social » à partir du récit d'un conseiller d'une mission locale $\left(T E \mathrm{n}^{\circ} 80\right)$; plus récemment, en 2008, Henri ECKERT et Virginie MORA analysent les trajectoires heurtées et incertaines d'insertion professionnelle des jeunes (TE $\left.\mathrm{n}^{\circ} 113\right)$. Plus généralement, la question du chômage et de ses effets sur les parcours demeure présente sur l'ensemble de la période, avec une attention accrue accordée au fil du temps aux chômeurs de longue durée, qui font 
l'objet de plusieurs articles à partir des années 1990. L'insertion professionnelle est également abordée via l'analyse des modes de recherche et d'obtention des emplois. Si dès 1982 une enquête de l'ANPE permet une analyse des modes d'embauche des salariés (BENARROCH, ESPINASSE, 1982, TE n ${ }^{\circ} 11$ ), c'est surtout au cours des décennies 2000 et 2010 que progresse le nombre d'articles sur cet objet, sur l'ANPE une nouvelle fois (LizÉ, 2000, $T E \mathrm{n}^{\circ}$ 83), les entreprises de travail temporaire dans l'automobile (Gorgeu, Mathieu, 2000, TE $\mathrm{n}^{\circ}$ 84), les petites annonces (Marchal, Torny, 2003, $T E \mathrm{n}^{\circ}$ 95), ou encore sur les modes d'obtention des emplois dans leur ensemble (DE LARQUIER, RIEUCAU, 2010,TE $\mathrm{n}^{\circ}$ 124). Ces publications renvoient plus largement à un champ de recherches sur les intermédiaires du marché du travail et sur l'analyse des conventions qui sous-tendent les pratiques de recrutement, champ ouvert par les travaux menés au Centre d'études de l'emploi à la fin des années 1990 (Bessy, EyMARD-DuVERnAY, 1997 ; EyMARD-DUVERnAY, MARCHAL, 1997).

À mesure que s'individualise la relation de travail, que déclinent les grandes instances de régulation collective et qu'émerge une mise en mobilité généralisée (CASTEL, 1995), les questions relatives aux carrières et aux mobilités se font plus présentes, et construisent un raisonnement à l'interface de l'individuel et du collectif. La thématique des carrières apparaît dans la revue avec des publications d'économistes, autour notamment de la question des carrières salariales (TRAVAIL ET EMPLOI n ${ }^{\circ} 54,1992$ ). Indissociable de la notion de carrière, la question de la mobilité apparaît également au tout début de la décennie 1990 sous l'angle de la mobilité interne aux entreprises ou aux organisations (PoDEvin, 1990, TE $\mathrm{n}^{\circ} 46$; MorEAU, 1992, TE $\mathrm{n}^{\circ} 53$ ) ou en lien avec le chômage à l'échelle européenne (LIANOS, NAGEL, 1995, TE n ${ }^{\circ} 62$ ). Quant à la dimension géographique de la mobilité, elle est notamment présente en filigrane de nombre d'articles consacrés aux chômeurs ou aux populations les plus fragiles.

Des travaux plus substantiels d'un point de vue sociologique ou économique, portant sur la compréhension d'un certain nombre de régularités ou de mécanismes explicatifs, se font plus nombreux à partir des années 1990. La connaissance s'affine ainsi progressivement : en 2008, Guillemette DE LARQUIER et Delphine REMILLON remettent en cause l'idée de l'apparition d'un « modèle général de mobilité », à rebours de nombre de discours entendus dans l'espace public ( $\left.T E \mathrm{n}^{\circ} 113\right)$; dans le même numéro, Thomas Amossé et Michel GolLAC relient l'évolution de la mobilité professionnelle à l'intensification du travail. Les analyses en termes de mobilité viennent croiser la dimension du genre et des professions, à l'image de l'article d'Anne LAMBERT et Delphine REMILLON (2018, $\left.T E \mathrm{n}^{\circ} 154\right)$, qui met en évidence la réduction en trompel'œil des inégalités de carrière entre stewards et hôtesses de l'air. Plus éloignée en apparence des thèmes du travail et de l'emploi, la mobilité sociale n'est guère présente dans la revue, à l'exception d'un article récent portant sur la mobilité sociale au sein de lignées d'actifs franciliens (REMILLON, LELIÈVRE, 2018, $T E \mathrm{n}^{\circ} 154$ ). 


\section{La flexibilisation de l'emploi par les entreprises : d'une gestion de crise à une gestion courante}

En quarante ans d'existence, la revue a mis en lumière les modifications profondes régissant la gestion des flux d'emplois par les entreprises et, plus généralement, les formes de recours à la flexibilité. Deux axes d'analyse majeurs apparaissent, l'un ayant trait à la gestion courante de la main-d'œuvre, et l'autre à sa gestion en temps de crise. Témoins des changements profonds intervenus sur le marché du travail, ces deux axes se confondent progressivement, les restructurations devenant un mode de gestion permanent de la main-d'œuvre et non plus seulement une réponse à la crise.

Le premier axe regroupe un ensemble d'articles portant sur la flexibilité en entreprise. Il se concentre d'abord sur la flexibilité comme mode d'organisation du travail (flexibilité des salaires, des horaires, de l'organisation, des emplois, des techniques), les embauches et les licenciements n'étant alors regardés que comme un moyen parmi d'autres pour l'entreprise d'ajuster à court terme sa production. Ces articles s'appuient sur des enquêtes conduites auprès des employeurs et retracent la diversité et l'évolution de leurs stratégies pour s'adapter à un environnement de plus en plus incertain. C'est ce que montre Pierre BoISARD (1994, TE $\left.\mathrm{n}^{\circ} 59\right)$ dans un article portant sur l'adaptation des horaires de travail aux fluctuations de la demande. Les entreprises ajustent également leur comportement au cadre législatif en vigueur, notamment avec le passage aux 35 heures, comme le montre Mathieu BunEL (2006, $\left.T E \mathrm{n}^{\circ} 106\right)$ dans un article portant sur les modes de flexibilité dans les établissements français. Il y remet en cause les typologies habituellement proposées, qui reposent sur les oppositions flexibilité interne/externe et flexibilité qualitative/quantitative dans lesquelles la nature des anticipations sur l'évolution de la demande joue un rôle central. Et il propose une typologie basée sur l'usage de la modulation, de la polyvalence et de l'intensité d'utilisation pour caractériser les différents types de gestion de la main-d'œuvre.

Dans les années 2000, la flexibilité est plus spécifiquement abordée sous l'angle de la facilité pour les entreprises à embaucher et débaucher, accompagnant en cela l'augmentation du recours aux contrats à durée déterminée (CDD). C'est dans ce contexte nouveau qu' apparaît le terme de « flexicurité » et la référence aux modèles des pays nordiques, pour lutter contre le chômage (cf. supra). La persistance d'un recours élevé aux CDD et l'apparition de la rupture conventionnelle donnent lieu à des articles étudiant les éventuelles substitutions à l'œuvre entre les différents modes de rupture des contrats de travail (BOURREAU-DuBOIs et al., 2011, TE $\mathrm{n}^{\circ}$ 126) et leurs implications au regard de la loi (SignoRETTO, 2015, TE $\mathrm{n}^{\circ}$ 142). Notons que les « difficultés de recrutement », qui occupent aujourd' hui une place de choix dans les débats, n'ont rien de nouveau. Elles sont mises en exergue dès 1991 (HENRY et al., 1991, TE n ${ }^{\circ} 49$ ) puis en 1999 (Du CREST, 1999, TE n ${ }^{\circ}$ 79), plus récemment en 2009 (dans le secteur de la pêche et en lien avec un dispositif d'insertion ; Gouzien, 2009, $T E \mathrm{n}^{\circ} 119$ ).

Le second axe traite de la question des flux d'emplois au regard des difficultés rencontrées par les entreprises (fusions, restructurations, faillites) et leurs conséquences 
en termes de licenciements et de réduction des effectifs. Cet axe peut être mis en rapport avec l'activité du ministère en charge du Travail, qui suit les conflits, plans sociaux et restructurations dans les entreprises, ce qui se traduit par des articles issus de commandes du ministère (FERnEZ, TonNEAU, 1987, TE $\mathrm{n}^{\circ} 33$ et TonNEAU, 1988, $T E \mathrm{n}^{\circ} 36-37$, sous l'angle de la productivité dans l'entreprise ; AUBERT, BEAUJOLINBellet, 2004, TE $\mathrm{n}^{\circ}$ 100, sous l'angle des mutations industrielles). Dans ce volant de travaux, on trouve majoritairement des monographies d'entreprises, qui visent à comprendre les décisions de suppression des sureffectifs (BEAUJOLIN, 1998, TE $\mathrm{n}^{\circ} 75$ ) puis ce qui devient un mode de gestion des entreprises (plans à répétition; AUBERT, BEAUJOLIN-BELLET, 2004, TE $\mathrm{n}^{\circ}$ 100). Les travaux pointent l'asymétrie d'information entre directions d'entreprises et représentants des salariés, l'intérêt qu'il peut y avoir pour les structures managériales à cacher l'information, mais aussi, dans certains cas, la volonté de l'entreprise de préserver son image par le dialogue (CAMPINOS-DUBERNET, 2003, $T E \mathrm{n}^{\circ}$ 95). À partir des années 2010, les travaux se focalisent davantage sur les conséquences de ces restructurations à répétition sur les individus, qu'ils restent au sein de l'entreprise - et sont ainsi soumis à une augmentation des risques psychosociaux (MESSAOUDI, 2011, TE $\mathrm{n}^{\circ}$ 126) ou qu'ils se retrouvent au chômage et soient incités à la création d'entreprise (DE LARQUIER, RIEUCAU, 2010,TE $\mathrm{n}^{\circ} 124$ ).

\section{Une transformation du tissu productif et de la structure professionnelle}

Enfin, des évolutions peuvent être soulignées concernant les métiers et secteurs d'activité examinés dans la revue. Bernard et Georgie ZARCA publient en 1979 un article portant sur «le cheminement professionnel des artisans », dont l'article de Caroline MAZAUd (2012, TE $\mathrm{n}^{\circ}$ 130) permet de mesurer la transformation, de même que les articles sur le monde ouvrier rendent compte des mutations qui l'affectent (IMBert, 1986, TE $\mathrm{n}^{\circ} 27$; COMBes, 1995, TE $\mathrm{n}^{\circ} 62$; RoupNEL-Fuentes, 2014, TE $\mathrm{n}^{\circ} 138$; TRANCHANT, 2018, TE $\mathrm{n}^{\circ} 155-156$ ). De manière symptomatique, le dernier de ces articles rend compte de la situation d'emploi des ouvriers de la logistique, activité située à la frontière des activités tertiaires. Les années 2000 constituent de fait un tournant avec une multiplication des monographies sur des activités de travail dans les services. Ont ainsi été publiés des articles sur les auxiliaires de vie, les infirmières ou, plus largement, les soignants, sur les centres d'appel, la grande distribution ou le travail en front office et, plus récemment, sur l'hôtellerie, les agents de guichet SNCF ou les hôtesses d'accueil et de caisse. Le secteur public n'est pas oublié avec un numéro thématique à dimension historique consacré aux mobilités des fonctionnaires (TRAVAIL ET EMPLOI $\mathrm{n}^{\circ}$ 127, 2011). Pris dans leur ensemble, ces articles constituent un bon traceur de l'évolution des secteurs d'activité et des marchés du travail, ils ont par exemple permis de suivre de près l'explosion du secteur des services à la personne. 
Que retenir au terme de ce panorama ? Sans céder à toute autocélébration malvenue, il semble possible de souligner un certain nombre d'éléments qui contribuent à l'originalité du regard porté par Travail et Emploi sur... le travail et l'emploi. Ce dernier se nourrit tout d'abord d'une place importante accordée aux enquêtes, depuis les grandes enquêtes de la statistique publique jusqu'aux enquêtes de terrain, ce qui fait de Travail et Emploi une revue résolument empirique, et c'est forte de cette assise qu'elle entend contribuer aux débats théoriques dans le champ. Tout au long de ces quatre décennies, la revue a par ailleurs publié des articles proposant des comparaisons internationales qui nourrissent la réflexion sur le cas français. Enfin, Travail et Emploi est une revue pluridisciplinaire, ce qui n'est somme toute peut-être pas si fréquent dans le champ des revues françaises en sciences sociales. En effet, si la revue s'est fait l'écho d'un certain nombre d'évolutions des champs de l'économie et de la sociologie du travail et de l'emploi, elle a ménagé une place singulière aux contributions provenant d'autres disciplines. C'est évidemment le cas des travaux d'histoire sociale ou du travail qui, bien que peu fréquents, ont toujours été présents dans les pages de Travail et Emploi (cf. l'article d'Anne-Sophie Bruno dans ce numéro anniversaire). De même, si les contributions de juristes sont restées très minoritaires, et en très grande majorité en provenance de deux laboratoires particulièrement engagés dans le dialogue interdisciplinaire - le laboratoire nantais Droit et changement social (DCS), et l'Institut de recherche sur l'entreprise et les relations professionnelles (Irerp) à Nanterre -, la revue a régulièrement traité de droit du travail. Quelques articles de politistes ont également été publiés, principalement dans le domaine des relations de travail et du syndicalisme. Le thème des conditions de travail et de la santé au travail a favorisé la publication d'articles venant de l'ergonomie et de la psychologie. La présence des ergonomes est particulièrement remarquable au début des années 2000, même s'ils sont déjà présents dans les années 1980, et on peut relever des contributions relevant de la psychodynamique du travail, sur les mêmes périodes. Ces présences reflètent l'attention portée par les comités de rédaction successifs à l'entretien de réflexions disciplinaires et pluridisciplinaires sur les enjeux de santé au travail, notamment à l'occasion de numéros spéciaux ${ }^{13}$.

L'ivresse des 40 ans ne doit cependant pas empêcher de relever un certain nombre d'angles morts et de thèmes relativement peu présents dans la revue. Peu d'articles abordent par exemple directement la thématique de la protection sociale, même dans ses aspects les plus en prise avec le travail et l'emploi, comme les retraites ou les minima sociaux liés à la situation professionnelle. Ces questions ne figurent dans la revue que de façon très secondaire (à l'exception notable du numéro de 2017 sur les marges de l'emploi et de la protection sociale, TRAVAIL ET EMPLOI $\mathrm{n}^{\circ} 149$ ). Cela reflète en partie la distinction avec d'autres revues (Revue française des affaires sociales, Retraite et société), mais ces thématiques pourraient davantage être abordées dans les pages de Travail et Emploi. C'est principalement sous l'angle des populations

13. Par exemple le numéro 94 en 2003 sur le dialogue entre ergonomes et sociologues et le numéro 129 en 2012 qui présente différents travaux menés dans le cadre du Collège d'expertise sur le suivi des risques psychosociaux au travail. 
qu'elles concernent de fait (pauvres, précaires, exclus ; jeunes, femmes, travailleurs clandestins), et de façon ponctuelle, que ces questions sont étudiées dans une période de quarante ans marquée par la récurrence, voire la permanence, de la « crise » comme contexte d'analyse. De la même manière, les questions liées aux migrations, ainsi qu'à la dimension ethnoraciale dans les mondes du travail, sont relativement peu présentes, comparativement à celles liées au genre notamment. De manière plus surprenante, des thèmes «classiques », centraux dans l'analyse du travail et de l'emploi, ont été relativement peu abordés dans la revue (comme l'intensification du travail), de même qu'il y a peu d'analyses croisant les classes sociales, la situation de travail et de revenu.

Nous aurions beau jeu d'affirmer que ces questions susciteront toute l'attention de Travail et Emploi au cours des prochaines décennies. Mais après tout, la fête passée et les regrets exprimés, que reste-t-il des anniversaires sinon des promesses pour l'avenir ?

\section{BIBLIOGRAPHIE}

\section{Articles et dossiers publiés dans Travail et Emploi}

Amable B., Françon B. (2015), «Quels effets des baisses de durée d'indemnisation sur la durée du chômage et le type d'emploi repris ? Le cas des lois Hartz», Travail et Emploi, $\mathrm{n}^{\circ} 142$, pp. 85-98.

Amossé T., Gollac M. (2008), «Intensité du travail et mobilité professionnelle », Travail et Emploi, n 113 , pp. 59-73.

Andolfatto D. (1997), « La syndicalisation à la CGT et à la CFDT, une étude locale », Travail et Emploi, $\mathrm{n}^{\circ} 71$, pp. 3-20.

Assouline M., Fodha M., Lemiale L., Zagamé P. (1997), « L'impact macro-économique d'une baisse des cotisations sociales employeurs sur les bas salaires », Travail et Emploi, $\mathrm{n}^{\mathrm{o}} 73$, pp. 41-52.

Aubert J.-P., BEAujolin-BelLet R. (2004), « Les acteurs de l'entreprise face aux restructurations : une délicate mutation », Travail et Emploi, n 100, pp. 99-112.

AUJARD J.-P., VolKOFF S. (1986), « Une analyse chiffrée des audiences syndicales », Travail et Emploi, $\mathrm{n}^{\circ} 30$, pp. 47-57.

BARALdi L., LAMOtTE B. (1998), « Négociation sur l'emploi : les logiques d'une transaction », Travail et Emploi, $\mathrm{n}^{\circ}$ 76, pp. 15-24.

BARANGer B. (1995), « "L'emploi et les relations sociales dans les PME". Compte rendu du colloque du 11 octobre $1994 »$, Travail et Emploi, n 63, pp. 89-99.

BARRAgan K., HARDY-Dubernet A.-C. (2005), « Le bilan de compétences : un espace de transition? », Travail et Emploi, n 103 , pp. 29-40.

BARREAU J., BROCHARD D. (2003), «Les politiques de rémunération des entreprises : écarts entre pratiques et discours », Travail et Emploi, n ${ }^{\circ}$ 93, pp. 45-59. 
BEAUD S. (1999), « Un cas de sauvetage social. Histoire d'un “jeune précaire” racontée par un conseiller de mission locale », Travail et Emploi, $\mathrm{n}^{\circ}$ 80, pp. 77-89.

Beaudouin V., Aucouturier A.-L. (1995), « Histoires d'insertion. Analyse lexicale de 580 récits de parcours de jeunes », Travail et Emploi, $\mathrm{n}^{\circ}$ 65, pp. 19-38.

BEAujolin R. (1998), «Les engrenages de la décision de réduction des effectifs », Travail et Emploi, $\mathrm{n}^{\circ}$ 75, pp. 19-32.

Benallah S., Domin J.-P. (2017), « Intensité et pénibilités du travail à l'hôpital. Quelles évolutions entre 1998 et 2013 ? », Travail et Emploi, $\mathrm{n}^{\circ}$ 152, pp. 5-31.

Benarroch F., EspinAsse J.-M. (1982), « Les salariés recrutés en 1980. Caractéristiques et modes d'embauche », Travail et Emploi, $\mathrm{n}^{\mathrm{0}} 11$, pp. 71-81.

BERCOT R. (2015), « Le devenir des femmes en chirurgie : la diversité des mondes de socialisation », Travail et Emploi, n ${ }^{\circ} 141$, pp. 37-52.

Berthet T., CONTER B. (2011), « Les changements d'instruments de la politique de l'emploi en Wallonie et en France », Travail et Emploi, n ${ }^{\circ} 125$, pp. 55-65.

Bethoux É. (2004), « Les comités d'entreprise européens en quête de légitimité », Travail et Emploi, n ${ }^{\circ} 98$, pp. 21-35.

Bevort A. (1995), « Compter les syndiqués, méthodes et résultats : la CGT, la CFDT, 1945$1990 »$, Travail et Emploi, $\mathrm{n}^{\circ}$ 62, pp. 40-58.

Bloch-London C., Coutrot T., Didry C., Michon F. (1999), « Découvrir la réduction et l'aménagement des temps de travail, la mise en œuvre des accords "Robien" dans douze petites et moyennes entreprises », Travail et Emploi, n ${ }^{\circ} 79$, pp. 89-109.

BoISARD P. (1980), «Avancées et limites de la recherche sur les effets de la réduction de la durée du travail », Travail et Emploi, $\mathrm{n}^{\circ} 3$, pp. 85-90.

BoISARD P. (1994), « L'adaptation des horaires de travail aux fluctuations de la demande. Les stratégies des entreprises », Travail et Emploi, $\mathrm{n}^{\circ}$ 59, pp. 18-30.

Bonafe-SchmitT J.-P. (1983), «Les nouveaux prud'hommes : un nouvel enjeu syndical ? », Travail et Emploi, n ${ }^{\circ} 18$, pp. 89-94.

Boni-Le GofF I. (2012), « “Ni un homme, ni une femme, mais un consultant.” Régimes de genre dans l'espace du conseil en management », Travail et Emploi, n ${ }^{\circ} 132$, pp. 21-34.

Bonvin J.-M., BADAN P. (2007), « La responsabilité sociale des entreprises à l'aune des restructurations : une étude de cas dans l'industrie des machines suisses », Travail et Emploi, $\mathrm{n}^{\mathrm{o}} 109$, pp. 59-68.

Bosch G., LaLlement M. (1991), « La négociation collective sur le temps de travail en France et en Allemagne », Travail et Emploi, n 49, pp. 31-45.

Boumahdi R., Lattes J.-M., Plassard J.-M. (2005), « Droit et économie de la discrimination sur le marché du travail », Travail et Emploi, $\mathrm{n}^{\circ}$ 104, pp. 55-68. 
Bourreau-Dubois C., Chaupain-Guillot S., Guillot O. (2011), «L'impact du risque prud'homal sur le recours aux contrats à durée déterminée : une analyse à partir des $D M M O$ », Travail et Emploi, n ${ }^{\circ} 126$, pp. 5-16.

BRIDGFORd J., STIRLING J. (1987), « Les relations professionnelles en Grande-Bretagne dans les années 80. Le défi au syndicalisme », Travail et Emploi, n 34, pp. 81-90.

Broudic P., EsPINASSE J.-M. (1980), « Les politiques de gestion de la main-d'œuvre », Travail et Emploi, $\mathrm{n}^{\circ}$ 6, pp. 7-13.

BRUCY G. (2001), « La doctrine de la CGT sur la formation des adultes : entre pragmatisme et lutte de classes (1945-1955) », Travail et Emploi, $\mathrm{n}^{\circ}$ 86, pp. 65-86.

BuÉ J., Rey F., RouX-Rossi D. (2013), « Le temps partiel moins attractif ? Nouvelles régulations temporelles dans deux secteurs de services », Travail et Emploi, n ${ }^{\circ}$ 134, pp. 59-74.

BuÉ J., RouX-Rossi D. (2002), «Salarié(e)s à temps partiel et réduction collective du temps de travail : la question du choix », Travail et Emploi, n ${ }^{\circ}$ 90, pp. 39-53.

Bughin E. (1982), « Négociations collectives et politique du SMIC. Un instrument d'appréciation », Travail et Emploi, $\mathrm{n}^{\circ} 14$, pp. 101-108.

BUNEL J. (1997), « Représentation patronale et représentativité des organisations patronales », Travail et Emploi, $\mathrm{n}^{\circ}$ 70, pp. 3-19.

Bunel M. (2006), «L'utilisation des modes de flexibilité par les établissements français », Travail et Emploi, $\mathrm{n}^{\mathrm{o}}$ 106, pp. 7-24.

Bureau M.-C., Sarfati F., Simha J., TuchSZIRER C. (2013), « L'expérimentation dans l'action publique. Usages, pratiques et jugements des acteurs », Travail et Emploi, $\mathrm{n}^{\circ} 135$, pp. 41-55.

BURGi N. (1986), « La grève des mineurs britanniques : un tournant », Travail et Emploi, $\mathrm{n}^{\circ} 30$, pp. 59-69.

Buscatto M. (2001), « La relation d'emploi à l'ombre du chômage : le cas de deux sociétés privées de service », Travail et Emploi, n ${ }^{\circ}$ 7, pp. 67-79.

CAHUC P. (2009), « Supprimer les entraves à l'activité et à la recherche d'emploi », Travail et Emploi, $\mathrm{n}^{\circ} 118$, pp. 61-86.

CAHUC P., Granier P. (1998), « La réduction du temps de travail : une solution pour l'emploi ? », Travail et Emploi, $\mathrm{n}^{\circ}$ 74, pp. 111-120.

CAmpinos-Dubernet M. (2003), « Des restructurations discrètes : reconstruire l'emploi face à la mondialisation », Travail et Emploi, $\mathrm{n}^{\circ}$ 95, pp. 41-57.

Cette G., Gubian A. (1998), «Les évaluations des effets sur l'emploi d'une réduction de la durée du travail : quelques remarques », Travail et Emploi, n 74, pp. 91-107.

CÉzArd M., Dussert F., Gollac M. (1992), « Taylor va au marché. Organisation du travail et informatique », Travail et Emploi, n ${ }^{\circ}$ 54, pp. 4-19.

Charvet M., Laurioux F., Lazuech G. (2016), « Quand la pénibilité du travail débarque. Les temps des pêcheurs et de leurs conjointes », Travail et Emploi, n ${ }^{\circ} 147$, pp. 53-75. 
Colin J.-F. (1981), « Le coût de la politique de l'emploi. Rétrospective 1973-1978 », Travail et Emploi, $\mathrm{n}^{\circ} 8$, pp 57-66.

Colin J.-F., Cros J.-C., Verdier É., Welcomme D. (1981), « Politiques d'emploi : la rupture de 1977. Éléments pour une analyse critique des politiques spécifiques d'emploi (1974-1980)», Travail et Emploi, $\mathrm{n}^{\circ}$ 10, pp. 9-22.

Combes M.-C. (1995), «Le déclin des professions ouvrières », Travail et Emploi, $\mathrm{n}^{\circ}$ 62, pp. 83-88.

Coutrot T. (1992), «L'intéressement : vers une nouvelle convention salariale ? », Travail et Emploi, n ${ }^{\circ} 53$, pp. 22-39.

Coutrot T. (1995), « Gestion de l'emploi et organisation du travail dans les entreprises innovantes. Une approche statistique des pratiques d'établissements », Travail et Emploi, $\mathrm{n}^{\circ}$ 64, pp. 79-87.

DAssa S. (1981), «La durée des grèves en France. Étude des fiches de conflit du travail de $1976 »$, Travail et Emploi, n ${ }^{\circ}$ 7, pp. 59-77.

Davoine L. (2005), « Le New Deal for Young People : des évaluations contrastées », Travail et Emploi, $\mathrm{n}^{\mathrm{o}} 101$, pp. 7-19.

DEAKIN S. (2007), « Restructurations et gouvernance d'entreprise en Grande-Bretagne : la vente de Rover », Travail et Emploi, n 109, pp. 51-58.

DeCouflé A.-C. (1983), « Sous-prolétariat et monde du travail », Travail et Emploi, $\mathrm{n}^{\circ} 16$, pp. 59-65.

DELFINI C., DEMAZIÈRE D. (2000), « Le traitement de l'offre d'emploi à l'ANPE : diversité des logiques d'intermédiation », Travail et Emploi, $\mathrm{n}^{\circ}$ 81, pp. 27-40.

DelteIL V. (2006), « Les comités d'entreprise européens : quelles perspectives ? », Travail et Emploi, $\mathrm{n}^{\circ} 106$, pp. 51-64.

Delteil V., DieuAide P. (2010), « Les comités d'entreprise européens dans l'UE élargie : entre outil de gestion et levier syndical », Travail et Emploi, $\mathrm{n}^{\circ} 123$, pp. 53-65.

DemaZiÈre D., Dubar C. (1996), « Récits d'insertion et mondes socio-professionnels. Analyse d'entretiens de jeunes peu diplômés et sortis de l'école en 1986 », Travail et Emploi, n 69 , pp. 55-69.

DivaY S. (2000), «L'aide à la recherche d'emploi : une activité en voie de professionnalisation ?», Travail et Emploi, $\mathrm{n}^{\circ} 81$, pp. 67-80.

Du CReSt A. (1999), « Le paradoxe persistant des difficultés de recrutement en période de chômage », Travail et Emploi, $\mathrm{n}^{0}$ 79, pp. 111-126.

Dupray A., Moullet S. (2015), « Le salaire moindre des femmes : une question d'individu ou de profession? », Travail et Emploi, $\mathrm{n}^{\circ}$ 144, pp. 81 -107.

ECKERT H., MORA V. (2008), « Formes temporelles de l'incertitude et sécurisation des trajectoires dans l'insertion professionnelle des jeunes », Travail et Emploi, n 113, pp. 31-57. 
ERHEL C. (1999), « Les politiques actives de l'emploi en France et en Suède. Une convergence récente de la structure des dépenses et des institutions ? », Travail et Emploi, $\mathrm{n}^{\circ}$ 79, pp 17-35. FERRACCI M., MARTIN F. (2013), «Évaluation par expérimentation aléatoire de dispositifs de reclassement innovants pour les demandeurs d'emploi », Travail et Emploi, n ${ }^{\circ}$ 135, pp. 15-26.

Fernez S., Tonneau D. (1987), « Productivité et emploi. Une relation complexe au sein de l'entreprise », Travail et Emploi, $\mathrm{n}^{\circ}$ 33, pp. 43-49.

Fremigacci F., TERracol A. (2014), « L'activité réduite en France : effet d'enfermement et effet tremplin », Travail et Emploi, n ${ }^{\circ} 139$, pp. 25-37.

FurJot D., FeuERBACh É. (1986), « Les conflits du travail en 1985 », Travail et Emploi, n 28 , pp. 7-22.

FurJot D., NoEl C. (1987), « La conflictualité en 1986. Bilan statistique et qualitatif », Travail et Emploi, $\mathrm{n}^{\circ} 34$, pp. 55-69.

FREYSSINET J. (1980), « Les lieux de gestion de la force de travail dans la stratégie des groupes industriels », Travail et Emploi, $\mathrm{n}^{\circ}$ 4, pp. 7-15.

Freyssinet J. (2009), «Flexibilité et sécurité : quelles stratégies d'acteurs ? », Travail et Emploi, n ${ }^{\mathrm{o}} 118$, pp. 113-121.

GARner H., MÉdA D., SENIK C. (2005), « Conciliation entre vie professionnelle et vie familiale, les leçons des enquêtes auprès des ménages », Travail et Emploi, n 102, p. 57-67.

Garonna P., Reboani P. (1991), « Politiques du temps de travail et système de relations professionnelles : l'expérience italienne », Travail et Emploi, ${ }^{\circ}$ 48, pp. 48-58.

GAZIER B. (2008), « Flexicurité et marchés transitionnels du travail : esquisse d'une réflexion normative », Travail et Emploi, $\mathrm{n}^{\mathrm{o}} 113$, pp. 117-128.

Giraud B., Marchand A., Penissat É. (2016), « Le sentiment de discrimination des représentants du personnel. Une étude à partir des données statistiques et monographiques liées à l'enquête REPONSE », Travail et Emploi, $\mathrm{n}^{\circ} 145$, p. 87-119.

Godinot X., JoIn-LAmberT L. (1984), «L'emploi des sous-prolétaires : constats et chances à saisir », Travail et Emploi, n ${ }^{\circ} 21$, pp. 35-48.

Gomel B., SERVERIN E. (2013), « L'expérimentation sociale aléatoire en France en trois questions », Travail et Emploi, ${ }^{\circ}$ 135, pp. 57-71.

Gontier G., BARON C., LABOuRIE-RACAPÉ A. (1981), «Quelques éléments sur la réinsertion professionnelle des femmes de plus de trente ans », Travail et Emploi, n ${ }^{\circ}$ 9, pp. 57-63.

Gorgeu A., Mathieu R. (2000), « Compétence et sélectivité du recrutement : l'exemple des usines de la filière automobile », Travail et Emploi, $\mathrm{n}^{\circ}$ 84, pp. 75-94.

Gouzien A. (2009), « Pénurie de main-d'œuvre et dispositifs localisés d'insertion dans les pêches maritimes en Bretagne », Travail et Emploi, n 119, pp. 23-35.

GRAu PINEDA C. (2007), « Le harcèlement moral au travail dans la législation espagnole : du risque psychosocial à l'obligation de sécurité de l'employeur », Travail et Emploi, $\mathrm{n}^{\mathrm{0}} 112$, pp. 49-61. 
GRIMAUD P. (2018), « L'extension du travail dominical dans le commerce. Vers une redéfinition sous tensions de la qualification des emplois », Travail et Emploi, $\mathrm{n}^{0} 155-156$, pp. 141-163.

GRIMAult S. (2008), «Sécurisation des parcours professionnels et flexicurité : analyse comparative des positions syndicales », Travail et Emploi, $\mathrm{n}^{\mathrm{o}} 113$, pp. 75-89.

GRISEZ J. (1982), « L'insertion professionnelle des stagiaires AFPA. Un aspect de l'évolution du rapport formation-emploi », Travail et Emploi, $\mathrm{n}^{\circ}$ 14, pp. 41-53.

Gubian A. (2000), « La réduction du temps de travail à mi-parcours : premier bilan des effets sur l'emploi », Travail et Emploi, n ${ }^{\circ} 83$, pp. 9-26.

HARDY-Dubernet A.-C. (1998), « La formation professionnelle continue dans les très petites entreprises : du devoir à l'obligation », Travail et Emploi, $\mathrm{n}^{0} 77, \mathrm{pp} .41-51$.

HenRiot-Olm C. (1997), « Allégement des charges sur les bas salaires. Stratégie de l'entreprise et effets sur l'emploi », Travail et Emploi, $\mathrm{n}^{\mathrm{0}}$ 73, pp. 53-66.

Henry B., Merle V., Weil N. (1991), «Difficultés de recrutement et gestion locale de l'emploi », Travail et Emploi, n’ 49, pp. 4-19.

Hingel A. J. (1985), « Le droit de retrait du poste de travail au Danemark », Travail et Emploi, $\mathrm{n}^{\mathrm{o}} 25$, pp. 41-54.

HoARAU C., JACQUIER J.-P. (1995), « Le comité d'entreprise européen : instance en devenir », Travail et Emploi, $\mathrm{n}^{\circ}$ 65, pp. 51-60.

IMBERT F. (1986), «Évolution du travail d'aiguillage et devenir des aiguilleurs », Travail et Emploi, n ${ }^{\circ} 27$, pp. 21-30.

JEFFERYS S. (2016), « Des syndicalistes britanniques toujours blacklistés ! L'anti-syndicalisme patronal au Royaume-Uni », Travail et Emploi, n 146, pp. 17-49.

KergoAt D. (1984), «Les femmes et le travail à temps partiel : une relation multiforme et complexe au temps travaillé », Travail et Emploi, $\mathrm{n}^{\mathrm{o}} 21$, pp. 7-21.

KESSLER F. (1990), « L'expertise en technologie en RFA : une analyse du contentieux », Travail et Emploi, $\mathrm{n}^{\circ} 43$, pp. 29-40.

Kosonen P. (1999), «Activation, incitations au travail et workfare dans quatre pays scandinaves », Travail et Emploi, $\mathrm{n}^{\mathrm{o}} 79$, pp. 1-15.

LABBÉ D. (1994), « Comités d'entreprise : les configurations électorales », Travail et Emploi, $\mathrm{n}^{\circ} 59$, pp. 64-73.

Labit A., Thoemmes J. (1995), «La "semaine de 4 jours" chez Volkswagen : un scénario original de sortie de crise ? », Travail et Emploi, n ${ }^{\circ}$ 64, pp. 5-22.

LAmbert A., Remillon D. (2018), « Une marche vers l'égalité professionnelle en trompel'œil. Disponibilité biographique et inégalités de carrière des hôtesses et stewards », Travail et Emploi, $\mathrm{n}^{\circ} 154$, pp. 5-41.

LARoQue G. (2009), « La Stratégie de l'OCDE pour l'emploi est-elle adaptée à la France ? », Travail et Emploi, $\mathrm{n}^{\mathrm{0}} 118$, pp. 55-60. 
LARQUiER G. (DE), REMILLON D. (2008), «Assiste-t-on à une transformation uniforme des carrières professionnelles vers plus de mobilité ? Une exploitation de l'enquête Histoire de vie », Travail et Emploi, $\mathrm{n}^{\mathrm{o}} 113$, pp. 13-30.

LARQuiER G. (DE), RIEUCAU G. (2010), « Trouver ou créer son emploi : compter sur soi, sur autrui ou sur les institutions ? », Travail et Emploi, $\mathrm{n}^{\circ}$ 124, pp. 43-55.

Lefebvre A., MÉDA D. (2008), «Performances nordiques et flexicurité : quelles relations ?», Travail et Emploi, n ${ }^{\circ} 113$, pp. 129-138.

LeFresne F., Sauviat C. (2009), « Mode de gouvernance et régimes de restructuration : une étude de cas », Travail et Emploi, n ${ }^{\circ} 117$, pp. 39-51.

Lianos F., NAGel O. (1995), « La libre circulation des chômeurs au sein de la communauté européenne : conditions et perspectives », Travail et Emploi, n ${ }^{\circ} 62$, pp. 72-75.

LIZÉ L. (2000), « Politiques de recrutement des entreprises et aides à l'emploi : quel rôle pour l'ANPE ? », Travail et Emploi, n 83, pp. 97-114.

MALLET L. (1989), « La détermination du sureffectif dans l'entreprise : démarche gestionnaire et construction sociale », Travail et Emploi, $\mathrm{n}^{\circ}$ 40, pp. 22-32.

Marchal E., Torny D. (2003), «Des petites aux grandes annonces : le marché des offres d'emploi depuis $1960 »$, Travail et Emploi, n $^{\circ}$ 95, pp. 59-72.

MAYERE A. (1983), « Revalorisation qualitative des emplois et substitution de jeunes travailleurs français à des travailleurs immigrés. Le cas d'une entreprise de collecte des ordures », Travail et Emploi, $\mathrm{n}^{\mathrm{o}} 17$, pp. 41-47.

Mazaud C. (2012), «Artisan, de l'homme de métier au gestionnaire », Travail et Emploi, $\mathrm{n}^{\mathrm{o}} 130$, pp. 9-20.

Messaoudi D. (2011), « La fusion-réorganisation dans un groupe financier. Une analyse des facteurs de risques psychosociaux », Travail et Emploi, $\mathrm{n}^{\circ} 126$, pp. 17-33.

MicheAu M. (1982), « Les comités de bassin d'emploi : bilan et questions », Travail et Emploi, $\mathrm{n}^{\mathrm{o}} 11$, pp. 9-26.

MinÉ M. (1999), « Approche juridique de la discrimination raciale au travail : une comparaison France-Royaume-Uni », Travail et Emploi, nº 80, pp. 91-107.

Moczall A. (2014), «Effets d'aubaine et de substitution d'un dispositif allemand de subvention salariale pour demandeurs d'emploi difficilement employables », Travail et Emploi, $\mathrm{n}^{\mathrm{o}} 139$, pp. 39-59.

Monchatre S. (2018), «Embaucher ou habiliter ? Recrutement et espaces de (non-)qualification dans l'hôtellerie-restauration », Travail et Emploi, n ${ }^{\circ}$ 155-156, pp. 93-114.

Moreau M.-A. (1992), «La mobilité des salariés dans les groupes de dimension communautaire : quelques réflexions à partir d'une analyse comparée », Travail et Emploi, $\mathrm{n}^{\circ} 53$, pp. 56-69.

Morin M.-L. (2003), « Compétences, mobilité et formation professionnelle, repères juridiques pour une réforme », Travail et Emploi, n ${ }^{\circ}$ 95, pp. 27-40. 
PASTRÉ O. (1983), « Taylorisme, productivité et crise du travail », Travail et Emploi, $\mathrm{n}^{\circ} 18$, pp. 43-70.

Pate F., Bez G., Koepr P., Tardieu M. (1980), « Les pactes nationaux pour l'emploi des jeunes », Travail et Emploi, n ${ }^{\circ}$ 6, pp. 15-61.

Pélisse J. (2000), « Le temps des négociations. Douze accords de réduction du temps de travail », Travail et Emploi, n ${ }^{\circ}$ 82, pp. 7-23.

PÉPIn M., Tonneau D. (1979), « Étude des conséquences des mesures de réduction de la durée du travail », Travail et Emploi, $\mathrm{n}^{\circ} 1$, pp. 7-22.

Podevin G. (1990), « Mobilité interne, promotions et renouvellement de la main-d'œuvre », Travail et Emploi, $\mathrm{n}^{\circ}$ 46, pp. 45-57.

REBÉRIOUX A. (2003), « Les marchés financiers et la participation des salariés aux décisions », Travail et Emploi, ${ }^{\circ}$ 93, pp. 25-43.

REMILlon D., Lelièvre É. (2018), « Positions et transmissions socioprofessionnelles des femmes et des hommes au sein de lignées franciliennes », Travail et Emploi, $\mathrm{n}^{\circ}$ 154, pp. 43-70.

RetTenbach B. (1979), « Diversité des formes juridiques de travail et restructuration des entreprises », Travail et Emploi, $\mathrm{n}^{\circ} 1$, pp. 23-27.

Roguet B., Salzberg L. (1991), «Dépenses actives et dépenses passives pour l'emploi de 1985 à $1989 »$, Travail et Emploi, n 48, pp. 75-79.

Roupnel-Fuentes M. (2014), « Carrières de licencié.e·s », Travail et Emploi, nº 138, pp. 19-36.

SAGLio J. (1986), « Hiérarchies salariales et négociations de classifications. France, 1900$1950 »$, Travail et Emploi, $\mathrm{n}^{\circ} 27$, pp. 7-19.

SCHMID G. (1995), « Le plein emploi est-il encore possible ? Les marchés du travail "transitoires" en tant que nouvelle stratégie dans les politiques d'emploi », Travail et Emploi, $\mathrm{n}^{\circ}$ 65, pp. 5-17.

Segrestin D. (1990), « Sur la représentation de l'entreprise en sociologie », Travail et Emploi, n 46 , pp. 23-29.

SignORETTO C. (2015), «Les pratiques des employeurs en matière de rupture du CDI : un nouveau regard sur les règles de protection de l'emploi », Travail et Emploi, n ${ }^{\circ}$ 142, pp. 69-83.

Simon J., Gourmelen M. (1979), « Enquête sur le devenir professionnel des jeunes ayant effectué un stage pratique en entreprise », Travail et Emploi, $\mathrm{n}^{\circ} 1$, pp. 65-74.

SouAmaA N. (2007), « La loi des huit heures : un projet d'Europe sociale ? (1918-1932)», Travail et Emploi, $\mathrm{n}^{\mathrm{O}} 110$, pp. 27-36.

StePHAN G. (2014), « Hétérogénéité sectorielle des effets d'un dispositif de subvention salariale sur les salaires et l'emploi en Allemagne », Travail et Emploi, n 139, pp. 61-74.

Supiot A. (1991), « L'application du droit du travail en Europe », Travail et Emploi, n 47 , pp. 4-13.

Tonneau D. (1988), « Productivité et emploi dans l'entreprise. Essai d'interprétation des approches actuelles », Travail et Emploi, $\mathrm{n}^{\mathrm{o}} 36-37, \mathrm{pp} .49-58$. 
ToRns T. (2008), « La place des femmes dans l'emploi en Espagne : les limites des politiques de conciliation vie familiale/vie professionnelle », Travail et Emploi, n ${ }^{\circ} 115$, pp. 59-70.

TRANCHANT L. (2018), « L'intérim de masse comme vecteur de disqualification professionnelle. Le cas des emplois ouvriers de la logistique », Travail et Emploi, ${ }^{\circ}$ 155-156, pp. 115-140.

TRAVAIL ET EMPLOI (1980), dossier « Le temps de travail », $\mathrm{n}^{\circ} 3$.

TRAVAIL ET EMPLOI (1985), dossier « L'expression directe des salariés », nº 24.

TRAVAIL ET EMPLOI (1989), dossier «L'évolution des formes d'emploi en : Italie, Espagne, France, Allemagne, Grande-Bretagne, Suède, États-Unis, Japon », nº 39.

TRAVAIL ET EMPLOI (1992), dossier « L'analyse économique des carrières salariales ", $\mathrm{n}^{\circ} 54$.

TRAVAIL ET EMPLOI (1993), dossier « La gestion prévisionnelle de l'emploi à l'épreuve des faits $», n^{\circ} 57-2$.

TRAVAIL ET EMPLOI (1996), dossier « Les relations sociales en entreprise : une approche nouvelle $», n^{\circ} 66$.

TRAVAIL ET EMPLOI (2003), dossier « Le travail aujourd'hui. Regards d'ergonomes et de sociologues », n 94.

TRAVAIL ET EMPLOI (2004), «Les nouvelles procédures communautaires/Marché du travail : d'un concept à l'autre/Relations professionnelles et lieux de décision/Vers la construction d'un nouveau système? », $\mathrm{n}^{\mathrm{o}} 100$.

TRAVAIL ET EMPLOI (2005), dossier «L'égalité femmes/hommes », nº 102.

TRAVAIL ET EMPLOI (2009), dossier «La réévaluation de la stratégie de l'OCDE pour l'emploi en 2006 : quels enseignements pour la France ? », nº 118.

TRAVAIL ET EMPLOI (2010), dossier « Multinationales françaises et relations d'emploi dans les pays d'Europe centrale et de l'Est », $\mathrm{n}^{\circ} 123$.

TRAVAIL ET EMPLOI (2011), dossier « Promotions et migrations administratives : histoire, ethnographie, approches croisées $\gg, \mathrm{n}^{\circ} 127$.

TRAVAIL ET EMPLOI (2012), dossier « Les risques psychosociaux au travail : d'une "question de société" à des questions scientifiques », nº 129.

TRAVAIL ET EMPLOI (2012), dossier « Représentativités syndicales, représentativités, patronales. Règles juridiques et pratiques sociales », n 131.

TRAVAIL ET EMPLOI (2014), dossier « Une crise sans précédent? Expériences et contestations des restructurations (I) », $\mathrm{n}^{\circ} 137$.

TRAVAIL ET EMPLOI (2014), dossier « Une crise sans précédent? Expériences et contestations des restructurations (II) », $\mathrm{n}^{\circ} 138$.

TRAVAIL ET EMPLOI (2014), dossier « Évaluation des politiques actives du marché du travail », $n^{\circ} 139$.

TRAVAIL ET EMPLOI (2015), dossier « Les institutions du travail : quelles réévaluations ? », n 142. 
TRAVAIL ET EMPLOI (2016), dossier « La discrimination syndicale en question : la situation en France $», n^{\circ} 145$.

TRAVAIL ET EMPLOI (2016), dossier « La discrimination syndicale en question : un panorama international $», \mathrm{n}^{\circ} 146$.

TRAVAIL ET EMPLOI (2016), dossier « Quand la pénibilité du travail s'invite à la maison », $\mathrm{n}^{\circ} 147$.

TRAVAIL ET EMPLOI (2017), dossier « Marges de l'emploi et protection sociale », $\mathrm{n}^{\circ} 149$.

TRAVAIL ET EMPLOI (2017), dossier « Genre et travail indépendant », $\mathrm{n}^{\circ} 150$.

TRAVAIL ET EMPLOI (2018), dossier «La Garantie jeunes : éléments d'évaluation et de comparaison internationale $», \mathrm{n}^{\circ} 153$.

Treutenaere M. (1979), « Travail et marché de l'emploi. Évolutions des inégalités », Travail et Emploi, $\mathrm{n}^{\circ} 2$, pp. 89-95.

TuCHSZirer C. (1986), «Les politiques de partage et d'aménagement du temps de travail en Belgique », Travail et Emploi, $\mathrm{n}^{\circ} 28$, pp. 45-56.

UbBiali G. (1999), « La reconversion des professionnels du syndicalisme », Travail et Emploi, $\mathrm{n}^{\circ} 80$, pp. 141-155.

VERDIER É. (1996), « L'insertion des jeunes “à la française” : vers un ajustement structurel ? », Travail et Emploi, n ${ }^{\circ}$ 69, pp. 37-54.

VILLEY O. (1980), « La main-d'œuvre étrangère et la crise en France », Travail et Emploi, $\mathrm{n}^{\mathrm{o}} 4$, pp. 83-92.

Zimmermann B. (2000), «Logiques de compétences et dialogue social », Travail et Emploi, $\mathrm{n}^{\mathrm{o}} 84$, pp. 5-19.

\section{Autres références bibliographiques}

AMossÉ T. (2020, à paraître), "Homo Statisticus. A History of France's General Public Statistical Infrastructure on Population Since 1950”, in Mennicken A., Salais R. (eds.), The New Politics of Numbers, London, Palgrave Macmillan.

Amossé T., Bloch-London C., WolfF L. (dir.) (2008), Les Relations sociales en entreprise. Un portrait à partir des enquêtes "Relations professionnelles et négociations d'entreprise » (REPONSE 1992-1993, 1998-1999 et 2004-2005), Paris, La Découverte.

Bessy C., Eymard-Duvernay F. (dir.) (1997), Les Intermédiaires du marché du travail, Paris, Presses universitaires de France, coll. « Les Cahiers du Centre d'études de l'emploi ».

Boussard V., Demazière D., Milburn P. (dir.) (2010), L'Injonction au professionnalisme. Analyses d'une dynamique plurielle, Rennes, Presses universitaires de Rennes.

CASTEL R. (1995), Les Métamorphoses de la question sociale. Une chronique du salariat, Paris, Fayard.

Fligstein N. (1990), The Transformation of Corporate Control, Cambridge (Mass.)/London, Harvard University Press. 
Champy F. (2012), La Sociologie des professions, $2^{\mathrm{e}}$ éd., Paris, Presses universitaires de France.

Chauvel L. (1998), Le Destin des générations. Structure sociale et cohortes en France au $X X^{e}$ siècle, Paris, Presses universitaires de France.

DemaZière D., GADÉA C. (dir.) (2009), Sociologie des groupes professionnels. Acquis récents et nouveaux défis, Paris, La Découverte.

EyMARD-Duvernay F., MARChAL E. (1997), Façons de recruter. Le jugement des compétences sur le marché du travail, Paris, Metailié.

GALLAND O. (2000), «L'allongement de la jeunesse en Europe », Revue de l'OFCE, $\mathrm{n}^{\circ} 72$, pp. 187-191.

Gollac M., VolKOFF S. (2010), « Mesurer le travail. Une contribution à l'histoire des enquêtes françaises dans ce domaine », Document de travail, n 127, Centre d'études de l'emploi.

Lоснак D. (1987), « Réflexions sur la notion de discrimination », Droit social, $\mathrm{n}^{\circ} 11$, pp. 778-790.

PÉLISSIER J. (dir.) (1999), Droit de l'emploi, 1999. Insertion, réinsertion, recrutement, contrats de travail..., Paris, Dalloz.

Peugny C., VAn de Velde C. (2013), « Repenser les inégalités entre générations », Revue française de sociologie, vol. 54, $\mathrm{n}^{\circ}$ 4, pp. 641-662.

THÉvENOT L. (1979), « Une jeunesse difficile : les fonctions sociales du flou et de la rigueur dans les classements », Actes de la recherche en sciences sociales, n ${ }^{\circ} 26-27$, pp. 3-18.

VIET V. (2006), « De la Revue française du Travail à la Revue française des Affaires sociales : une histoire passée en revue », Revue française des affaires sociales, $\mathrm{n}^{\circ}$ 4, pp. 47-57.

ZARCA B., ZARCA G. (1979), Le Cheminement professionnel des artisans : le travail familial et la réussite de l'entreprise. Rapport final, Paris, Crédoc. 